\title{
DRINFELD DOUBLE OF DEFORMED QUANTUM ALGEBRAS
}

\author{
ZHAOBING FAN AND JUNJING XING
}

\begin{abstract}
We provide a deformation, $\mathfrak{f}_{\beta}$, of Lusztig algebra $\mathbf{f}$. Various quantum algebras in literatures, including half parts of two-parameter quantum algebras, quantum superalgebras, and multi-parameter quantum algebras/superalgebras, are all specializations of $\mathfrak{f}_{\beta}$. Moreover, $\mathfrak{f}_{\beta}$ is isomorphic to Lusztig algebra $\mathbf{f}$ up to a twist. As a consequence, half parts of those quantum algebras are isomorphic to Lusztig algebra $\mathbf{f}$ over a big enough ground field up to certain twists. We further construct the entire algebra $\mathbf{U}_{\beta, \xi}$ by Drinfeld double construction. As special cases, above quantum algebras all admit a Drinfeld double construction under certain assumptions.
\end{abstract}

\section{Contents}

1. Introduction

2. Deformation of half part of quantum algebras 3

3. Drinfeld double of $\mathfrak{f}_{\beta}$

4. Specializations of $\mathbf{U}_{\beta, \xi}$

References $\quad 22$

\section{INTRODUCTION}

In FL, the first author and Li constructed an algebra by using mixed perverse sheaves on representation varieties of a quiver, and showed that the algebra is isomorphic to the negative part of two-parameter quantum algebras defined in literatures. As a byproduct, the half part of two-parameter quantum algebras is isomorphic to that of Lusztig algebra $\mathbf{f}$ defined in L10, Chapter 1] after a twist involving only the second parameter. Later on, in [CFLW], Clark-Fan-Li-Wang showed that under certain assumptions, the half part of quantum superalgebras is isomorphic to its non-super analogue after a twist. These results provided a novel way to study problems related to two-parameter quantum algebras/quantum superalgebras, for example, in [C17, the author showed that knot invariants from quantum algebra and its super analogy are the same using the twist in [CFLW].

Date: November 5, 2019.

2010 Mathematics Subject Classification. 17B37, 20G42, 81R50.

Key words and phrases. quantum algebras, deformation, Drinfeld double. 
It's a natural question how far the above results can be generalized to? In this paper, we provide a deformation $\mathfrak{f}_{\beta}$ of the Lusztig algebra $\mathbf{f}$ associated with a Cartan datum $(I, \cdot)$ over $\mathbb{F}$, where $\mathbb{F}$ is a ground field containing $\mathbb{Q}(v)$ and $\alpha, \beta: \mathbb{N}[I] \times \mathbb{N}[I] \rightarrow \mathbb{F}$ are two multiplicative bilinear forms. Various quantum algebras in literatures, including two-parameter quantum algebras in [FL, quantum superalgebras in [CHW], multi-parameter quantum algebras in HPR and multi-parameter quantum superalgebras in $\mathrm{KKO}$, are all specializations of $\mathfrak{f}_{\beta}$. We further show the following theorem (Theorem 1 in Section 2.2).

Theorem A For all homogenous elements $x, y \in \mathfrak{f}_{\beta}$, let $x * y=\gamma(|x|,|y|)$ $x y$, then

$$
\left(\mathfrak{f}_{\beta}, *\right) \simeq \mathbf{f} \otimes_{\mathbb{Q}(v)} \mathbb{F}
$$

where $\gamma: \mathbb{N}[I] \times \mathbb{N}[I] \rightarrow \mathbb{F}$ is a multiplicative bilinear form satisfying (5).

As special cases, Theorem A shows that half parts of those quantum algebras are isomorphic to Lusztig algebra $\mathbf{f}$ after certain twists. This partially answers the earlier question we raised. Theorem A also indicates that there might be some relationships among the representations of those quantum algebras. In fact, in FLL, Fan-Li-Lin proved that the categories of weight modules of those quantum algebras are all equivalent.

We note that the twisted $*$-product on $\mathfrak{f}_{\beta}$ is a variant of the deformations defined in [FL, Section 4] (resp. [CFLW, Section 2]) relating one-parameter quantum groups to its two-parameter analogy (resp. super analogy). Surprisingly, the choice of $\gamma(-,-)$ in Theorem A is not unique. So Theorem A not only generalizes the results in [FL] (resp. [CFLW]) to other quantum algebras, but also discovers new phenomena for two-parameter quantum algebras (resp. quantum superalgebras).

Drinfeld [D87] built a braided Hopf algebra out of any finite-dimensional Hopf algebra with the invertible antipode, which is called "the Drinfeld double construction", providing the solution of the Yang-Baxter equation. In recent years, Drinfeld doubles have been studied by various authors as a useful tool of recovering the entire quantum groups from half parts. Xiao X97 got a complete realization of quantum groups by applying the reduced form of the Drinfeld double of a Hall algebra to the generic composition algebra. Benkart-Witherspoon [BW] characterized two-parameter quantum groups of type A as the Drinfeld double of the certain Hopf subalgebras with respect to a skew-dual paring. Following this work, Bergeron-Gao-Hu BGH] provided the Drinfeld double construction of two-parameter quantum groups of types B, C and D. Moreover, Hu-Rosso-Zhang [HRZ], Hu-Zhang [HZ] and Gao-Hu-Zhang [GHZ] gave the Drinfeld double construction of two-parameter quantum affine algebras $U_{r, s}\left(\widehat{\mathfrak{s l}_{n}}\right), U_{r, s}\left(C_{n}^{(1)}\right)$ and $U_{r, s}\left(G_{2}^{(1)}\right)$, respectively. 
In this paper, we study the entire quantum group of the algebra $\mathfrak{f}_{\beta}$ by constructing a Drinfeld double $\mathbf{U}_{\beta, \xi}$, where $\xi: \mathbb{N}[I] \times \mathbb{N}[I] \rightarrow \mathbb{F}$ is a symmetric multiplicative bilinear form. As special cases, two-parameter quantum algebras, quantum superalgebras, and multi-parameter quantum algebras/superalgebras all admit a Drinfeld double construction.

This paper is organized as follows. In Section 2, we provide the deformation $\mathfrak{f}_{\beta}$ of Lusztig algebra $\mathbf{f}$ in [L10, Chapter 1] and show that $\mathfrak{f}_{\beta}$ is isomorphic to the ordinary quantum algebra up to a twist. In Section 3, we construct the Drinfeld double, $\mathbf{U}_{\beta, \xi}$, of the algebra $\mathfrak{f}_{\beta}$ as a Hopf algebra. In Section 4 , we provide various specializations of $\mathbf{U}_{\beta, \xi}$ which are shown to be isomorphic to various quantum algebras in literatures.

Acknowledgements. The first author thanks Zongzhu Lin for earlier discussions which motivate this project. We thank Yiqiang Li, Sean Clark and Weiqiang Wang for fruitful collabrations, which provided some examples for this project. Z. Fan is partially supported by the NSF of China grant 11671108, the NSF of Heilongjiang Province grant LC2017001 and the Fundamental Research Funds for the central universities GK2110260131.

\section{Deformation of HALF PART OF QUANTUM ALGEBRAS}

In this section, we provide a deformation, $\mathfrak{f}_{\beta}$, of the Lusztig algebra in [L10, Chapter 1] depending on two multiplicative bilinear forms $\alpha, \beta$ : $\mathbb{N}[I] \times \mathbb{N}[I] \rightarrow \mathbb{F}$ and study some examples of $\mathfrak{f}_{\beta}$.

2.1. Deformation of the Lusztig algebra. Let us fix a Cartan datum $(I, \cdot)$. We simply write $d_{i}=\frac{i \cdot i}{2}$ and $a_{i j}=2 \frac{i \cdot j}{i \cdot i}$. Let $v$ be an indeterminate. We set $v_{i}=v^{d_{i}}$ for all $i \in I$. Given a field $\mathbb{F}$ containing $\mathbb{Q}(v)$, let $\alpha, \beta$ : $\mathbb{N}[I] \times \mathbb{N}[I] \rightarrow \mathbb{F}$ be two multiplicative bilinear forms satisfying the following properties:

(i) $\beta(i, j) \alpha(j, i)=\beta(j, i) \alpha(i, j), \quad \forall i, j \in I$;

(ii) $\beta(i, j) \beta(j, i)=1, \quad \forall i, j \in I$, we further assume that $\beta(i, i)=1$.

Let $\mathcal{f}$ be the free unital associative $\mathbb{F}$-algebra generated by $\left\{\theta_{i}, i \in I\right\}$. The algebra $\mathcal{f}$ is an $\mathbb{N}[I]$-graded algebra if we set the grading of $\theta_{i}$ to be $i$. For any homogenous element $x \in \mathcal{f}$, we denote by $|x|$ the grading of $x$.

We define an algebra structure on $\mathcal{f} \otimes$ 角 by

$$
\left(x_{1} \otimes x_{2}\right)\left(y_{1} \otimes y_{2}\right)=v^{-\left|y_{1}\right| \cdot\left|x_{2}\right|} \beta\left(\left|x_{2}\right|,\left|y_{1}\right|\right) x_{1} y_{1} \otimes x_{2} y_{2},
$$

for any homogenous elements $x_{2}, y_{1} \in$ '牛.

Let $r:$ 角 $\rightarrow$ 午 $\otimes$ 角 be the $\mathbb{F}$-algebra homomorphism such that

$$
r\left(\theta_{i}\right)=\theta_{i} \otimes 1+1 \otimes \theta_{i}, \quad \forall i \in I .
$$

Proposition 1. There is a unique symmetric bilinear form $(-,-): \mathcal{f} \times \mathcal{f} \rightarrow$ $\mathbb{F}$ such that

(a) $(1,1)=1$ and $\left(\theta_{i}, \theta_{j}\right)=\delta_{i j}\left(1-v_{i}^{-2}\right)^{-1}, \quad \forall i, j \in I$;

(b) $\left(x, y^{\prime} y^{\prime \prime}\right)=\left(r(x), y^{\prime} \otimes y^{\prime \prime}\right), \quad \forall x, y^{\prime}, y^{\prime \prime} \in$ 争; 
(c) $\left(x^{\prime} x^{\prime \prime}, y\right)=\left(x^{\prime} \otimes x^{\prime \prime}, r(y)\right), \quad \forall x^{\prime}, x^{\prime \prime}, y \in$ 午.

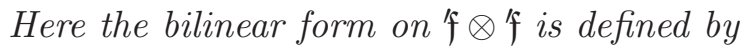

$$
\left(x_{1} \otimes x_{2}, y_{1} \otimes y_{2}\right)=\alpha\left(\left|x_{1}\right|,\left|x_{2}\right|\right)\left(x_{1}, y_{1}\right)\left(x_{2}, y_{2}\right),
$$

for any homogenous elements $x_{1}, x_{2}, y_{1}, y_{2} \in$ 午.

Proof. The proof for Proposition 13 in [FL works through if we replace $t^{2\left(2 \delta_{i j} \Omega_{i i}-\Omega_{i j}\right)}$ and $t^{\Omega_{j i}-\Omega_{i j}}$ by corresponding bilinear forms $\alpha(i, j)$ and $\beta(i, j)$, respectively. For part (c), one need use the property (i).

Let $\mathfrak{J}$ be the radical of the bilinear form $(-,-)$. It is clear that $\mathfrak{J}$ is a two-sided ideal of $\mathcal{f}$. Denote the quotient algebra of $\mathcal{f}$ by $\mathfrak{J}$ by

$$
\mathfrak{f}_{\beta}=\mathfrak{⿰} / \mathfrak{J} \text {. }
$$

For any $i \in I$, let $r_{i}\left(\operatorname{resp} .{ }_{i} r\right):$ ' $\rightarrow$ 角 be the unique linear map satisfying the following properties:

$$
\begin{aligned}
& r_{i}(1)=0, r_{i}\left(\theta_{j}\right)=\delta_{i j}, \forall j \in I \text { and } r_{i}(x y)=v^{-i \cdot|y|} \beta(i,|y|) r_{i}(x) y+x r_{i}(y) ; \\
& { }_{i} r(1)=0,{ }_{i} r\left(\theta_{j}\right)=\delta_{i j}, \forall j \in I \text { and }{ }_{i} r(x y)={ }_{i} r(x) y+v^{-i \cdot|x|} \beta(|x|, i) x_{i} r(y) .
\end{aligned}
$$

By an induction on $|x|$, we can show that $r(x)=r_{i}(x) \otimes \theta_{i}$ (resp. $r(x)=$ $\left.\theta_{i} \otimes_{i} r(x)\right)$ plus other terms. By this property, we have the following lemma.

Lemma 1. (a) For any $x, y \in$ 午, we have

$$
\left(y \theta_{i}, x\right)=\alpha(|y|, i)\left(y, r_{i}(x)\right)\left(\theta_{i}, \theta_{i}\right) \text { and }\left(\theta_{i} y, x\right)=\alpha(i,|y|)\left(\theta_{i}, \theta_{i}\right)\left(y,{ }_{i} r(x)\right) .
$$

(b) For any $i \in I$, the linear maps ${ }_{i} r, r_{i}:$ 午 $\rightarrow$ 午 send $\mathfrak{J}$ to itself.

By using the same argument as that for Lemma 1.2.15 in [L10], we have the following lemma.

Lemma 2. If a homogenous element $x \in \mathfrak{f}_{\beta}$ satisfies that $r_{i}(x)=0$ (resp. ${ }_{i} r(x)=0$ ) for all $i \in I$, then $x=0$.

For any $n \in \mathbb{N}$ and $c \in \mathbb{F}$, we set

$$
[n]_{c}=\frac{c^{n}-c^{-n}}{c-c^{-1}}, \quad[n]_{c}^{!}=\prod_{k=1}^{n}[k]_{c} \quad \text { and } \quad\left[\begin{array}{l}
n \\
k
\end{array}\right]_{c}=\frac{[n]_{c}^{!}}{[k]_{c}^{!}[n-k]_{c}^{!}} .
$$

Let

$$
\theta_{i}^{(n)}=\frac{\theta_{i}^{n}}{[n]_{v_{i}}^{!}}
$$

By using $\left(1 \otimes \theta_{i}\right)\left(\theta_{i} \otimes 1\right)=v_{i}^{-2}\left(\theta_{i} \otimes 1\right)\left(1 \otimes \theta_{i}\right)$ and the quantum binomial formula in [L10, Section 1.3.5], we have the following lemma.

Lemma 3. We have ${ }_{i} r\left(\theta_{i}^{(n)}\right)=v_{i}^{-(n-1)} \theta_{i}^{(n-1)}$ for any $n \in \mathbb{N}$ and $i \in I$.

Proposition 2. The generators $\theta_{i}$ of $\mathfrak{f}_{\beta}$ satisfy the following relations.

$$
\sum_{k+k^{\prime}=1-a_{i j}}(-1)^{k} \beta(i, j)^{-k} \theta_{i}^{(k)} \theta_{j} \theta_{i}^{\left(k^{\prime}\right)}=0, \quad \forall i \neq j \in I .
$$


Proof. For simplicity, we write

$$
D_{i j}:=\sum_{k+k^{\prime}=1-a_{i j}}(-1)^{k} \beta(i, j)^{-k} \theta_{i}^{(k)} \theta_{j} \theta_{i}^{\left(k^{\prime}\right)}, \quad \forall i \neq j \in I .
$$

By Lemma 2, we only need to show that ${ }_{l} r\left(D_{i j}\right)=0$ for any $l \in I$. It is clear that

$$
{ }_{l} r\left(D_{i j}\right)=0, \quad \text { if } l \neq i, j .
$$

By Lemma 3 and the definition of ${ }_{i} r$, we have

$$
\begin{aligned}
& { }_{i} r\left(\theta_{i}^{(k)} \theta_{j} \theta_{i}^{\left(k^{\prime}\right)}\right)={ }_{i} r\left(\theta_{i}^{(k)} \theta_{j}\right) \theta_{i}^{\left(k^{\prime}\right)}+v^{-i \cdot(k i+j)} \beta(k i+j, i) v_{i}^{-\left(k^{\prime}-1\right)} \theta_{i}^{(k)} \theta_{j} \theta_{i}^{\left(k^{\prime}-1\right)} \\
= & v_{i}^{-(k-1)} \theta_{i}^{(k-1)} \theta_{j} \theta_{i}^{\left(k^{\prime}\right)}+v^{-i \cdot(k i+j)} \beta(j, i) v_{i}^{-\left(k^{\prime}-1\right)} \theta_{i}^{(k)} \theta_{j} \theta_{i}^{\left(k^{\prime}-1\right)} \\
= & v_{i}^{-(k-1)} \theta_{i}^{(k-1)} \theta_{j} \theta_{i}^{\left(k^{\prime}\right)}+v_{i}^{-k} \beta(j, i) \theta_{i}^{(k)} \theta_{j} \theta_{i}^{\left(k^{\prime}-1\right)} .
\end{aligned}
$$

So ${ }_{i} r\left(D_{i j}\right)$ is equal to

$$
\begin{aligned}
& \sum_{1 \leq k \leq 1-a_{i j}}(-1)^{k} v_{i}^{-(k-1)} \beta(i, j)^{-k} \theta_{i}^{(k-1)} \theta_{j} \theta_{i}^{\left(k^{\prime}\right)} \\
& +\sum_{0 \leq k \leq-a_{i j}}(-1)^{k} v_{i}^{-k} \beta(i, j)^{-(k+1)} \theta_{i}^{(k)} \theta_{j} \theta_{i}^{\left(k^{\prime}-1\right)} \\
& =\sum_{0 \leq k \leq-a_{i j}}(-1)^{(k+1)} v_{i}^{-k} \beta(i, j)^{-(k+1)} \theta_{i}^{(k)} \theta_{j} \theta_{i}^{\left(k^{\prime}-1\right)} \\
& +\sum_{0 \leq k \leq-a_{i j}}(-1)^{k} v_{i}^{-k} \beta(i, j)^{-(k+1)} \theta_{i}^{(k)} \theta_{j} \theta_{i}^{\left(k^{\prime}-1\right)} .
\end{aligned}
$$

By comparing the exponents of $v_{i}$ and $\beta(i, j)$, we have

$$
{ }_{i} r\left(D_{i j}\right)=0 \text {. }
$$

By Lemma 3 and the definition of ${ }_{j} r$ again, we have

$$
{ }_{j} r\left(\theta_{i}^{(k)} \theta_{j} \theta_{i}^{\left(k^{\prime}\right)}\right)=v^{-k j \cdot i} \beta(k i, j) \theta_{i}^{(k)} \theta_{i}^{\left(k^{\prime}\right)} .
$$

So ${ }_{j} r\left(D_{i j}\right)$ is equal to

$$
\begin{aligned}
& \sum_{k+k^{\prime}=1-a_{i j}}(-1)^{k} v^{-k j \cdot i} \theta_{i}^{(k)} \theta_{i}^{\left(k^{\prime}\right)} \\
= & \sum_{k+k^{\prime}=1-a_{i j}}(-1)^{k} v_{i}^{-k a_{i j}}\left[\begin{array}{c}
1-a_{i j} \\
k
\end{array}\right]_{v_{i}} \theta_{i}^{\left(1-a_{i j}\right)} .
\end{aligned}
$$

By Section 1.3.4 in [L10,

$$
\sum_{k+k^{\prime}=1-a_{i j}}(-1)^{k} v_{i}^{-k a_{i j}}\left[\begin{array}{c}
1-a_{i j} \\
k
\end{array}\right]_{v_{i}}=0 .
$$

So we have

$$
{ }_{j} r\left(D_{i j}\right)=0
$$


Proposition follows (2), (3) and (44).

2.2. Relation to the Lusztig algebra. Recall the definition of $\beta(-,-)$ from the Section 2.1. We assume that there exits a multiplicative bilinear form $\gamma: \mathbb{N}[I] \times \mathbb{N}[I] \rightarrow \mathbb{F}$ satisfying the following property:

$$
\gamma(i, j)=\gamma(j, i) \beta(j, i), \quad \forall i, j \in I .
$$

We define a new multiplication " $*$ " on $\mathfrak{f}_{\beta}$ as follows.

$$
x * y=\gamma(|x|,|y|) x y, \quad \text { for all homogenous elements } x, y \in \mathfrak{f}_{\beta} .
$$

Recall that $\mathbf{f}$ is Lusztig algebra defined in [L10, Chapter 1] associated to a Cartan datum $(I, \cdot)$. Let $\mathbf{f}_{\mathbb{F}}=\mathbf{f} \otimes_{\mathbb{Q}(v)} \mathbb{F}$.

Theorem 1. If there exists $\gamma(-,-)$ satisfying (5), then the map $\eta:\left(\mathfrak{f}_{\beta}, *\right) \rightarrow$ $\mathbf{f}_{\mathbb{F}}$ sending $\theta_{i}$ to $\theta_{i}$ for all $i \in I$ is an algebra isomorphism.

Proof. By using (5) and Proposition 2, the algebra $\left(\mathfrak{f}_{\beta}, *\right)$ is generated by $\theta_{i}, i \in I$ and subjects to the following relations:

$$
D_{i j}^{\prime}:=\sum_{k+k^{\prime}=1-a_{i j}}(-1)^{k}\left[\begin{array}{c}
1-a_{i j} \\
k
\end{array}\right]_{v_{i}} \theta_{i}^{* k} * \theta_{j} * \theta_{i}^{* k^{\prime}}=0 .
$$

Thus,

$$
\eta\left(D_{i j}^{\prime}\right)=\sum_{k+k^{\prime}=1-a_{i j}}(-1)^{k}\left[\begin{array}{c}
1-a_{i j} \\
k
\end{array}\right]_{v_{i}} \theta_{i}^{k} \theta_{j} \theta_{i}^{k^{\prime}}=0 .
$$

This finishes the proof.

Remark 1. In general, the choice of $\gamma(-,-)$ satisfying (5) is not unique if it exists. Therefore the twist in Theorem 1 is not unique.

2.3. Examples. In this subsection, we shall give some examples of $\mathfrak{f}_{\beta}$. Although the results for Examples 1 and 2 have been found in [FL and [CFLW], respectively, by Remark 1, the twists in Examples 1 and 2 are not unique. We shall provide a new twist for two-parameter quantum algebras (resp. quantum superalgebras). To the best of our knowledge, results for Examples 3, 4 and 5 are new in literatures.

Example 1. We briefly review the two-parameter quantum algebra $\mathfrak{f}_{v, t}$ defined in $[\mathrm{FL}$, where $t$ is the second indeterminate.

For a fixed Cartan datum $(I, \cdot)$, let $\Omega=\left(\Omega_{i j}\right)_{i, j \in I}$ be an integer matrix satisfying that

(a) $\Omega_{i i} \in \mathbb{Z}_{>0}, \Omega_{i j} \in \mathbb{Z}_{\leq 0}$ for all $i \neq j \in I$;

(b) $\frac{\Omega_{i j}+\Omega_{j i}}{\Omega_{i i}} \in \mathbb{Z}_{\leq 0}$ for all $i \neq j \in I$;

(c) the greatest common divisor of all $\Omega_{i i}$ is equal to 1 ;

(d) $i \cdot j=\Omega_{i j}+\Omega_{j i}, \quad \forall i, j \in I$. 
The two-parameter quantum algebra $\mathfrak{f}_{v, t}$ associated to $\Omega$ is the associative $\mathbb{Q}(v, t)$-algebra generated by $\theta_{i}, \forall i \in I$ and subjects to the following relations.

$$
\sum_{k+k^{\prime}=1-a_{i j}}(-1)^{k}\left[\begin{array}{c}
1-a_{i j} \\
k
\end{array}\right]_{v_{i}} t^{k\left(\Omega_{i j}-\Omega_{j i}\right)} \theta_{i}^{k} \theta_{j} \theta_{i}^{k^{\prime}}=0, \quad \forall i \neq j \in I .
$$

We set $\mathbb{F}=\mathbb{Q}(v, t)$ and

$$
\beta(i, j)=t^{\Omega_{j i}-\Omega_{i j}}, \quad \alpha(i, j)=t^{2\left(2 \delta_{i j} \Omega_{i i}-\Omega_{i j}\right)}, \quad \forall i, j \in I .
$$

We further set $\gamma(i, j)=t^{\Omega_{i j}-2 \delta_{i j} \Omega_{i i}}, \forall i, j \in I$. Under these settings, Properties (i), (ii) in Section 2.1 and (5) hold, and the construction in Section 2.1 turns into the construction in [FL, Section 3]. Under the specialization (6), $\mathfrak{f}_{\beta}$ is exactly the algebra $\mathfrak{f}_{v, t}$. By Theorem 1 $\left(\mathfrak{f}_{v, t}, *\right)$ is isomorphic to $\mathbf{f} \otimes_{\mathbb{Q}(v)} \mathbb{Q}(v, t)$, where $x * y=\gamma(|x|,|y|) x y$. This result has been found in FL. We note if we set

$$
\gamma(i, j)=v^{i \cdot j} t^{\Omega_{i j}}, \quad \forall i, j \in I,
$$

the result in Theorem 1 still holds.

Example 2. Recall the definition of the quantum superalgebra $\mathfrak{f}_{v, \mathbf{i}}$ from CHW. We assume that $(I, \cdot)$ is a bar-consistent Cartan super datum. By bar-consistent, we mean that $d_{i}$ is odd if and only if $i$ is odd. Let $\mathcal{P}: I \rightarrow \mathbb{Z}_{2}$ be the parity function such that $\mathcal{P}(i)=1$ if $i$ is odd and 0 otherwise. We set $t=\mathbf{i}$, the complex number such that $\mathbf{i}^{2}=-1$, and $q=v^{-1} t$.

The quantum superalgebra $\mathfrak{f}_{v, \mathbf{i}}$ associated to $(I, \cdot)$ is the associative $\mathbb{Q}(v, \mathbf{i})$ algebra generated by $\theta_{i}, \forall i \in I$ and subjects to the following relations.

$$
\sum_{k+k^{\prime}=1-a_{i j}}(-1)^{k}\left[\begin{array}{c}
1-a_{i j} \\
k
\end{array}\right]_{v_{i}} t^{k i \cdot j+2 k \mathcal{P}(i) \mathcal{P}(j)} \theta_{i}^{k} \theta_{j} \theta_{i}^{k^{\prime}}=0, \quad \forall i \neq j \in I .
$$

We set $\mathbb{F}=\mathbb{Q}(v, \mathbf{i})$ and

$$
\beta(i, j)=t^{i \cdot j} t^{2 \mathcal{P}(i) \mathcal{P}(j)}, \quad \alpha(i, j)=1, \quad \forall i, j \in I .
$$

In particular, $\beta(i, i)=1$. We further fix an order " $<$ " on $I$ and set

$$
\gamma(i, j)= \begin{cases}t^{i \cdot j} & \text { if } j<i \\ t^{d_{i}} & \text { if } j=i \\ t^{2 \mathcal{P}(i) \mathcal{P}(j)} & \text { if } j>i\end{cases}
$$

Under these settings, Properties (i), (ii) in Section 2.1 and (5) hold, and the construction in Section 2.1 turns into the construction in [CHW]. Moreover, we have

$$
\mathfrak{f}_{\beta}=\mathfrak{f}_{v, \mathbf{i}} .
$$

By Theorem 1. $\left(\mathfrak{f}_{v, \mathbf{i}}, *\right)$ is isomorphic to $\mathbf{f} \otimes_{\mathbb{Q}(v)} \mathbb{Q}(v, \mathbf{i})$, where $x * y=$ $\gamma(|x|,|y|) x y$. This result has been found in [CFLW]. If we set

$$
\gamma(i, j)= \begin{cases}t^{2 i \cdot j} & \text { if } j<i \\ t^{d_{i}} & \text { if } j=i \\ t^{i \cdot j} t^{2 \mathcal{P}(i) \mathcal{P}(j)} & \text { if } j>i\end{cases}
$$


the result in Theorem 1 still holds.

Example 3. Recall the definition of the multi-parameter quantum algebra $\mathfrak{f}_{v, \mathbf{q}}$ from [HPR. For a fixed Cartan datum $(I, \cdot)$, let $A=\left(a_{i j}\right)_{i, j \in I}$ be an associated generalized Cartan matrix and $q_{i j}, \forall i, j \in I$, be indeterminates over $\mathbb{Q}$ such that $q_{i j} q_{j i}=q_{i i}^{a_{i j}}$. We set $\mathbf{q}=\left(q_{i j}\right)_{i, j \in I}$ and further assume that

$$
q_{i i}=v_{i}^{-2}, \quad \forall i \in I \text {. }
$$

The multi-parameter quantum algebra $\mathfrak{f}_{v, \mathbf{q}}$ associated to $(I, \cdot)$ is the associative $\mathbb{Q}\left(v, q_{i j}^{1 / 2}\right)$-algebra generated by $\theta_{i}, \forall i \in I$ and subjects to the following relations.

$$
\sum_{k+k^{\prime}=1-a_{i j}}(-1)^{k}\left[\begin{array}{c}
1-a_{i j} \\
k
\end{array}\right]_{v_{i}} v^{-k i \cdot j} q_{i j}^{-k} \theta_{i}^{k} \theta_{j} \theta_{i}^{k^{\prime}}=0, \quad \forall i \neq j \in I .
$$

We set $\mathbb{F}=\mathbb{Q}\left(v, q_{i j}^{1 / 2}\right)$ and

$$
\beta(i, j)=v^{i \cdot j} q_{i j}, \quad \alpha(i, j)=q_{i j}, \quad \gamma(i, j)=q_{i i}^{\delta_{i j}} q_{j i}^{1 / 2}, \quad \forall i, j \in I .
$$

In particular, $\beta(i, i)=1$. Under the settings in (8),

$$
\mathfrak{f}_{\beta}=\mathfrak{f}_{v, \mathbf{q}}
$$

By Theorem 1, $\left(\mathfrak{f}_{v, \mathbf{q}}, *\right)$ is isomorphic to $\mathbf{f} \otimes_{\mathbb{Q}(v)} \mathbb{Q}\left(v, q_{i j}^{1 / 2}\right)$ under the assumption (77), where $x * y=\gamma(|x|,|y|) x y$.

Example 4. Recall the definition of the multi-parameter quantum superalgebra $\mathfrak{f}_{\mathbf{s}, \mathbf{p}}$ from [KKO, Section 2]. For a fixed bar-consistent super Cartan datum $(I, \cdot)$, let $\mathbf{s}:=\left\{s_{i j}\right\}_{i, j \in I}$ and $\mathbf{p}:=\left(\left\{p_{i j}\right\}_{i, j \in I},\left\{p_{i}\right\}_{i \in I}\right)$ be families of invertible elements of a commutative ring $\mathbb{Q}\left(p_{i}^{1 /\left(2 d_{i}\right)}, s_{i j}, p_{i j}\right)$ such that

$$
p_{i j}^{2}=p_{i}^{2 a_{i j}}, \quad\left(p_{i j} p_{j i}\right) /\left(s_{i j} s_{j i}\right)=p_{i}^{2 a_{i j}}, \quad \text { and } \quad p_{i i} / s_{i i}=p_{i}^{2}, \forall i, j \in I .
$$

The condition (9) implies

$$
p_{i}^{2 a_{i j}}=p_{j}^{2 a_{j i}}, \quad\left(s_{i j} s_{j i}\right)^{2}=1, \quad \text { and } \quad s_{i j} s_{j i}=p_{i j}^{-1} p_{j i} .
$$

We further assume that

$$
p_{i}=v_{i} h_{i} \quad \text { such that } h_{i}^{2}=1, \quad \forall i \in I .
$$

The multi-parameter quantum superalgebra $\mathfrak{f}_{\mathbf{s}, \mathbf{p}}$ associated to $(I, \cdot)$ is the associative $\mathbb{Q}\left(v^{1 / 2}, s_{i j}, p_{i j}\right)$-algebra generated by $\theta_{i}, \forall i \in I$ and subjects to the following relations.

$$
\sum_{k+k^{\prime}=1-a_{i j}}(-1)^{k}\left[\begin{array}{c}
1-a_{i j} \\
k
\end{array}\right]_{v_{i}} s_{j i}^{k} p_{j i}^{-k} v_{i}^{k a_{i j}} \theta_{i}^{k} \theta_{j} \theta_{i}^{k^{\prime}}=0, \quad \forall i \neq j \in I .
$$

We set $\mathbb{F}=\mathbb{Q}\left(v^{1 / 2}, s_{i j}, p_{i j}\right)$ and

$$
\beta(i, j)=s_{j i}^{-1} p_{j i} v_{i}^{-a_{i j}}, \quad \alpha(i, j)=s_{i j} p_{i j}^{-1}, \quad \forall i, j \in I .
$$


In particular, $\beta(i, i)=1$. We further fix an order " $<$ " on $I$ and set

$$
\gamma(i, j)= \begin{cases}s_{i j}^{-1} h_{i}^{a_{i j}} s_{j i} & \text { if } j<i \\ s_{i i} & \text { if } j=i \\ p_{i j} p_{j}^{-a_{j i}} s_{j i}^{-1} & \text { if } j>i\end{cases}
$$

Under the settings in (12),

$$
\mathfrak{f}_{\beta}=\mathfrak{f}_{\mathbf{s}, \mathbf{p}}
$$

By Theorem $1,\left(\mathfrak{f}_{\mathbf{s}, \mathbf{p}}, *\right)$ is isomorphic to $\mathbf{f} \otimes_{\mathbb{Q}(v)} \mathbb{Q}\left(v^{1 / 2}, s_{i j}, q_{i j}\right)$ under the assumption (11), where $x * y=\gamma(|x|,|y|) x y$.

Example 5. Recall the definition of the multi-parameter quantum superalgebra $\mathfrak{f}_{\tilde{\mathbf{s}}, \tilde{\mathbf{p}}}$ from [KKO, Section 3]. For a fixed bar-consistent super Cartan datum $(I, \cdot)$, let $\tilde{\mathbf{s}}:=\left\{\tilde{s}_{i j}\right\}_{i, j \in I}$ and $\tilde{\mathbf{p}}:=\left\{\tilde{p}_{i}\right\}_{i \in I}$ be families of invertible elements of a commutative ring $\mathbb{Q}\left(\tilde{p}_{i}^{1 /\left(2 d_{i}\right)}, \tilde{s}_{i j}\right)$ such that

$$
\tilde{s}_{i j} \tilde{s}_{j i}=\tilde{p}_{i}^{-a_{i j}}, \quad \text { and } \quad \tilde{s}_{i i}=\tilde{p}_{i}^{-1}, \forall i, j \in I .
$$

We further assume that

$$
\tilde{p}_{i}=v_{i}^{2}, \quad \forall i \in I .
$$

The multi-parameter quantum superalgebra $\mathfrak{f}_{\tilde{\mathbf{s}}, \tilde{\mathbf{p}}}$ associated to $(I, \cdot)$ is the associative $\mathbb{Q}\left(v, \tilde{s}_{i j}\right)$-algebra generated by $\theta_{i}, \forall i \in I$ and subjects to the following relations.

$$
\sum_{k+k^{\prime}=1-a_{i j}}(-1)^{k}\left[\begin{array}{c}
1-a_{i j} \\
k
\end{array}\right]_{v_{i}}\left(\tilde{s}_{i j} \tilde{p}_{i}^{a_{i j} / 2}\right)^{-k} \theta_{i}^{k} \theta_{j} \theta_{i}^{k^{\prime}}=0, \quad \forall i \neq j \in I .
$$

We set $\mathbb{F}=\mathbb{Q}\left(v, \tilde{s}_{i j}\right)$ and

$$
\beta(i, j)=\tilde{s}_{i j}\left(\tilde{p}_{i}\right)^{a_{i j} / 2}, \quad \alpha(i, j)=\tilde{s}_{i j}, \quad \forall i, j \in I .
$$

In particular, $\beta(i, i)=1$. We further fix an order " $<$ " on $I$ and set

$$
\gamma(i, j)= \begin{cases}\tilde{s}_{j i} & \text { if } j<i \\ \tilde{s}_{i i}^{3} & \text { if } j=i \\ \tilde{p}_{i}^{-a_{i j} / 2} & \text { if } j>i\end{cases}
$$

Under the settings in (14),

$$
\mathfrak{f}_{\beta}=\mathfrak{f}_{\tilde{\mathbf{s}}, \tilde{\mathbf{p}}}
$$

By Theorem 1, $\left(\mathfrak{f}_{\tilde{\mathbf{s}}, \tilde{\mathbf{p}}}, *\right)$ is isomorphic to $\mathbf{f} \otimes_{\mathbb{Q}(v)} \mathbb{Q}\left(v, \tilde{s}_{i j}\right)$ under the assumption (13), where $x * y=\gamma(|x|,|y|) x y$.

\section{DRinfeld Double of $\mathfrak{f}_{\beta}$}

3.1. We review briefly the Drinfeld double construction in X97. For a Hopf algebra $A$, we shall denote by $\Delta_{A}, \varepsilon_{A}$ and $S_{A}$ its comultiplication, counit and antipode, respectively. 
Definition 1. XX7, Section 2.2] Given $\mathbb{F}$-Hopf algebras $A$ and $B$, a skewHopf pairing of $A$ and $B$ is a bilinear form $\psi: A \times B \rightarrow \mathbb{F}$ satisfying

(a) $\psi(1, y)=\varepsilon_{B}(y), \quad \psi(x, 1)=\varepsilon_{A}(x) ;$

(b) $\psi\left(x, y^{\prime} y^{\prime \prime}\right)=\psi\left(\Delta_{A}(x), y^{\prime} \otimes y^{\prime \prime}\right)$;

(c) $\psi\left(x^{\prime} x^{\prime \prime}, y\right)=\psi\left(x^{\prime} \otimes x^{\prime \prime}, \Delta_{B}^{\mathrm{op}}(y)\right)$;

(d) $\psi\left(S_{A}(x), y\right)=\psi\left(x, S_{B}^{-1}(y)\right), \quad \forall x, x^{\prime}, x^{\prime \prime} \in A, y, y^{\prime}, y^{\prime \prime} \in B$,

where $\psi\left(x^{\prime} \otimes x^{\prime \prime}, y^{\prime} \otimes y^{\prime \prime}\right)=\psi\left(x^{\prime}, y^{\prime}\right) \psi\left(x^{\prime \prime}, y^{\prime \prime}\right)$ and $\Delta_{B}^{\mathrm{op}}(y)=\sum y_{2} \otimes y_{1}$ if $\Delta_{B}(y)=\sum y_{1} \otimes y_{2}$.

Proposition 3. X97, Proposition 2.4] Let $\psi: A \times B \rightarrow \mathbb{F}$ be a skewHopf pairing. Then $A \otimes B$ has a Hopf algebra structure. Moreover, for any $x, x^{\prime} \in A, y, y^{\prime} \in B$, the algebra structure on $A \otimes B$ is defined by

(i) $\quad(x \otimes 1)\left(x^{\prime} \otimes 1\right)=x x^{\prime} \otimes 1 ;$

(ii) $(1 \otimes y)\left(1 \otimes y^{\prime}\right)=1 \otimes y y^{\prime}$;

(iii) $\quad(x \otimes 1)(1 \otimes y)=x \otimes y$;

(iv) $(1 \otimes y)(x \otimes 1)=\sum \psi\left(x_{1}, S_{B}\left(y_{1}\right)\right) x_{2} \otimes y_{2} \psi\left(x_{3}, y_{3}\right)$,

where $\Delta_{A}^{2}(x)=\sum x_{1} \otimes x_{2} \otimes x_{3}$ and $\Delta_{B}^{2}(y)=\sum y_{1} \otimes y_{2} \otimes y_{3}$.

And the comultiplication $\Delta_{A \otimes B}$, counit $\varepsilon_{A \otimes B}$ and antipode $S_{A \otimes B}$ of $A \otimes B$ are given as follows.

$$
\begin{aligned}
& \Delta_{A \otimes B}(x \otimes y)=\sum\left(x_{1} \otimes y_{1}\right) \otimes\left(x_{2} \otimes y_{2}\right), \\
& \varepsilon_{A \otimes B}(x \otimes y)=\varepsilon_{A}(x) \varepsilon_{B}(y), \\
& S_{A \otimes B}(x \otimes y)=\left(1 \otimes S_{B}(y)\right)\left(S_{A}(x) \otimes 1\right), \quad \forall x \in A, y \in B,
\end{aligned}
$$

where $\Delta_{A}(x)=\sum x_{1} \otimes x_{2}$ and $\Delta_{B}(y)=\sum y_{1} \otimes y_{2}$.

3.2. The Hopf algebras $\widehat{\mathfrak{f}^{+}}$and $\widehat{\mathfrak{f}^{-}}$. Let $\mathfrak{H}\left(\right.$ resp. $\left.\mathfrak{H}^{\prime}\right)$ be the commutative $\mathbb{F}$-algebra generated by $K_{i}^{ \pm 1}, J_{i}^{ \pm 1}\left(\operatorname{resp} . K_{i}^{\prime \pm 1}, J_{i}^{\prime \pm 1}\right.$ ) for all $i \in I$ and subject to the relations $K_{i} K_{i}^{-1}=1, J_{i} J_{i}^{-1}=1\left(\operatorname{resp} . K_{i}^{\prime} K_{i}^{\prime-1}=1, J_{i}^{\prime} J_{i}^{\prime-1}=1\right)$.

Let $\xi: \mathbb{N}[I] \times \mathbb{N}[I] \rightarrow \mathbb{F}$ be a symmetric multiplicative bilinear form such that $\xi(i, i)^{1 / 2} \in \mathbb{F}, \forall i \in I$.

Recall that $\beta(-,-)$ is a multiplicative bilinear form defined in Section 2.1. We set

$$
\langle i, j\rangle=v^{-i \cdot j} \beta(i, j) \xi(j, i), \quad \forall i, j \in I,
$$

which is also a multiplicative bilinear form on $\mathbb{N}[I] \times \mathbb{N}[I]$.

To distinguish, we shall write $\hat{\mathfrak{f}}^{+}$(resp. $\mathcal{f}^{-}$) the free $\mathbb{F}$-algebra generated by $E_{i}\left(\right.$ resp. $F_{i}$ ), for all $i \in I$. We denote the algebra isomorphism by $\iota^{+}$: ' $\rightarrow$ ' $^{+}$with $\iota^{+}\left(\theta_{i}\right)=E_{i}$ and the algebra anti-isomorphism by $\iota^{-}:$' $^{-} \rightarrow$ ' $^{-}$ with $\iota^{-}\left(\theta_{i}\right)=F_{i}$. 
We set $\widehat{\mathfrak{f}^{+}}=\mathfrak{f}^{+} \rtimes \mathfrak{H}$ and $\widehat{\mathfrak{f}^{-}}=\mathfrak{f}^{-} \rtimes \mathfrak{H}^{\prime}$, where the $\mathfrak{H}$-action (resp. $\mathfrak{H}^{\prime}-$ action) on $\mathfrak{\mathcal { f }}^{+}$(resp. $\mathfrak{f}^{-}$) is defined by

$$
\begin{gathered}
K_{i} \cdot E_{j}:=K_{i} E_{j} K_{i}^{-1}=\langle i, j\rangle E_{j}, \quad J_{i} \cdot E_{j}:=J_{i} E_{j} J_{i}^{-1}=\xi(j, i) E_{j}, \\
K_{i}^{\prime} \cdot F_{j}:=K_{i}^{\prime} F_{j} K_{i}^{\prime-1}=\langle j, i\rangle \xi(i, j)^{-1} F_{j}, \quad J_{i}^{\prime} \cdot F_{j}:=J_{i}^{\prime} F_{j} J_{i}^{\prime-1}=F_{j}, \forall i, j \in I .
\end{gathered}
$$

Moreover, the graded structure on ${ }^{\prime} \mathfrak{f}^{+}\left(\right.$resp. ${ }^{\prime} \mathfrak{f}^{-}$) can be extended to $\widehat{\mathfrak{f}}^{+}$ (resp. $\widehat{\mathfrak{f}^{-}}$) by setting $\left|K_{i}\right|=\left|K_{i}^{\prime}\right|=0$ and $\left|J_{i}\right|=\left|J_{i}^{\prime}\right|=0$ for all $i \in I$.

The Hopf algebra structure $\left(\Delta_{+}, \varepsilon_{+}, S_{+}\right)\left(\operatorname{resp} . \quad\left(\Delta_{-}, \varepsilon_{-}, S_{-}\right)\right)$on $\widehat{\mathfrak{f}^{+}}$ (resp. $\widehat{\mathfrak{f}^{-}}$) is given as follows.

$$
\begin{array}{cl}
\Delta_{+}\left(E_{i}\right)=E_{i} \otimes J_{i}+K_{i} \otimes E_{i}, & \Delta_{+}\left(K_{i}^{ \pm 1}\right)=K_{i}^{ \pm 1} \otimes K_{i}^{ \pm 1}, \\
\Delta_{-}\left(F_{i}\right)=J_{i}^{\prime} \otimes F_{i}+F_{i} \otimes K_{i}^{\prime}, & \Delta_{-}\left(K_{i}^{ \pm \pm 1}\right)=K_{i}^{ \pm \pm 1} \otimes K_{i}^{\prime \pm 1}, \\
\Delta_{+}\left(J_{i}^{ \pm 1}\right)=J_{i}^{ \pm 1} \otimes J_{i}^{ \pm 1}, & \Delta_{-}\left(J_{i}^{ \pm 1}\right)=J_{i}^{ \pm 1} \otimes J_{i}^{ \pm 1}, \\
\varepsilon_{+}\left(K_{i}^{ \pm 1}\right)=\varepsilon_{+}\left(J_{i}^{ \pm 1}\right)=1, & \varepsilon_{+}\left(E_{i}\right)=0, \\
\varepsilon_{-}\left(K_{i}^{\prime \pm 1}\right)=\varepsilon_{-}\left(J_{i}^{\prime \pm 1}\right)=1, & \varepsilon_{-}\left(F_{i}\right)=0, \\
S_{+}\left(E_{i}\right)=-K_{i}^{-1} E_{i} J_{i}^{-1}, & S_{+}\left(K_{i}^{ \pm 1}\right)=K_{i}^{\mp 1}, \quad S_{+}\left(J_{i}^{ \pm 1}\right)=J_{i}^{\mp 1}, \\
S_{-}\left(F_{i}\right)=-J_{i}^{\prime-1} F_{i} K_{i}^{\prime-1}, & S_{-}\left(K_{i}^{ \pm \pm 1}\right)=K_{i}^{\prime \mp 1}, \quad S_{-}\left(J_{i}^{\prime \pm 1}\right)=J_{i}^{\prime \mp 1} .
\end{array}
$$

3.3. Skew Hopf pairing. For any $\nu \in \mathbb{Z}[I]$ and $j \in I$, we define $\mathbb{F}$-linear $\operatorname{maps}{ }_{j} \mathcal{S}, \mathcal{J}_{\nu}$ and $\mathcal{K}_{\nu}$ from $\widehat{\mathfrak{f}^{-}}$to itself by

$$
\begin{aligned}
& \mathcal{K}_{\nu}\left(K_{\tau_{1}}^{\prime} x J_{\tau_{2}}^{\prime}\right)=\left\langle\nu, \tau_{1}\right\rangle\langle\nu,|x|\rangle K_{\tau_{1}}^{\prime} x J_{\tau_{2}}^{\prime}, \\
& \mathcal{J}_{\nu}\left(K_{\tau_{1}}^{\prime} x J_{\tau_{2}}^{\prime}\right)=\xi\left(\tau_{1}, \nu\right) \xi(|x|, \nu) K_{\tau_{1}}^{\prime} x J_{\tau_{2}}^{\prime}, \quad \forall \tau_{1}, \tau_{2} \in \mathbb{Z}[I], x \in \mathfrak{f}^{-}, \\
& { }_{j} \mathcal{S}\left(K_{\tau_{1}}^{\prime} J_{\tau_{2}}^{\prime}\right)=0, \quad{ }_{j} \mathcal{S}\left(F_{i}\right)=\delta_{i j} \xi(i, j), \quad \text { and } \\
& { }_{j} \mathcal{S}(x y)={ }_{j} \mathcal{S}(x) \mathcal{J}_{j}(y)+\mathcal{K}_{j}(x)_{j} \mathcal{S}(y) .
\end{aligned}
$$

It is clear that $\mathcal{K}_{\nu}$ and $\mathcal{J}_{\nu}$ are well defined. We now check that ${ }_{j} \mathcal{S}$ is also well defined. The commutative relations on $\mathfrak{H}^{\prime}$ are trivial. By the definition of ${ }_{l} \mathcal{S}$, for any $i, j, l \in I$, we have

$$
\begin{aligned}
& { }_{l} \mathcal{S}\left(K_{i}^{\prime} F_{j}\right)=\delta_{j l}\langle j, i\rangle \xi(j, l) K_{i}^{\prime}=\langle j, i\rangle \xi(i, j)^{-1}{ }_{l} \mathcal{S}\left(F_{j} K_{i}^{\prime}\right), \\
& { }_{l} \mathcal{S}\left(J_{i}^{\prime} F_{j}\right)=\delta_{j l} \xi(j, l) J_{i}^{\prime}={ }_{l} \mathcal{S}\left(F_{j} J_{i}^{\prime}\right) .
\end{aligned}
$$

This shows ${ }_{l} \mathcal{S}$ is well defined. We note that $\left|{ }_{l} \mathcal{S}(x)\right|=|x|-l$ if $|x|_{l} \neq 0$.

Lemma 4. The assignment $E_{j} \mapsto\left(v_{j}^{-1}-v_{j}\right)^{-1}{ }_{j} \mathcal{S}, K_{\nu} \mapsto \mathcal{K}_{\nu}$ and $J_{\nu} \mapsto \mathcal{J}_{\nu}$ defines an algebra anti-homomorphism $\rho^{+}: \widehat{\mathfrak{f}^{+}} \rightarrow \operatorname{Hom}_{\mathbb{F}}\left(\widehat{\left(\mathfrak{f}^{-}\right.}, \widehat{\mathfrak{f}^{-}}\right)$.

Proof. For any $i, j \in I, \tau_{1}, \tau_{2} \in \mathbb{Z}[I]$ and $x \in^{\prime} \mathfrak{f}^{-}$, we have

$$
\begin{aligned}
& { }_{j} \mathcal{S}\left(\mathcal{K}_{i}\left(K_{\tau_{1}}^{\prime} x J_{\tau_{2}}^{\prime}\right)\right)=\left\langle i, \tau_{1}\right\rangle\langle i,|x|\rangle\left\langle j, \tau_{1}\right\rangle K_{\tau_{1} j}^{\prime} \mathcal{S}(x) J_{\tau_{2}}^{\prime}, \quad \text { and } \\
& \mathcal{K}_{i}\left({ }_{j} \mathcal{S}\left(K_{\tau_{1}}^{\prime} x J_{\tau_{2}}^{\prime}\right)\right)=\left\langle i, \tau_{1}\right\rangle\langle i,|x|-j\rangle\left\langle j, \tau_{1}\right\rangle K_{\tau_{1} j}^{\prime} \mathcal{S}(x) J_{\tau_{2}}^{\prime} .
\end{aligned}
$$

This shows that $\rho^{+}\left(K_{i} E_{j}\right)=\langle i, j\rangle \rho^{+}\left(E_{j} K_{i}\right)$. 
Similarly, for any $i, j \in I, \tau_{1}, \tau_{2} \in \mathbb{Z}[I]$ and $x \in^{\prime} \mathfrak{f}^{-}$, we have

$$
\begin{aligned}
& { }_{j} \mathcal{S}\left(\mathcal{J}_{i}\left(K_{\tau_{1}}^{\prime} x J_{\tau_{2}}^{\prime}\right)\right)=\left\langle j, \tau_{1}\right\rangle \xi(|x|, i) \xi\left(\tau_{1}, i\right) K_{\tau_{1}}^{\prime}{ }_{j} \mathcal{S}(x) J_{\tau_{2}}, \quad \text { and } \\
& \mathcal{J}_{i}\left({ }_{j} \mathcal{S}\left(K_{\tau_{1}}^{\prime} x J_{\tau_{2}}^{\prime}\right)\right)=\left\langle j, \tau_{1}\right\rangle \xi(|x|-j, i) \xi\left(\tau_{1}, i\right) K_{\tau_{1} j}^{\prime} \mathcal{S}(x) J_{\tau_{2}}^{\prime} .
\end{aligned}
$$

This shows that $\rho^{+}\left(J_{i} E_{j}\right)=\xi(j, i) \rho^{+}\left(E_{j} J_{i}\right)$. The other defining relations for $\widehat{\mathfrak{f}}^{+}$are straightforward.

We define a bilinear form $\phi: \widehat{:}^{+} \times \widehat{\mathfrak{f}}^{-} \rightarrow \mathbb{F}$ by

$$
\phi(x, y)=\varepsilon_{-}\left(\rho^{+}(x) y\right), \forall x \in \widehat{\mathfrak{f}^{+}}, y \in \widehat{\mathfrak{f}^{-}} .
$$

The bilinear form $\phi$ is well defined. It is clear that $\phi(x, y)=0$ if $x, y$ are homogenous with different gradings.

Moreover, for any $\nu_{1}, \nu_{2}, \tau_{1}, \tau_{2} \in \mathbb{Z}[I]$ and any $x \in \mathfrak{f}^{+}, y \in \mathfrak{f}^{-}$, we have

$$
\phi\left(K_{\nu_{1}} x J_{\nu_{2}}, K_{\tau_{1}}^{\prime} y J_{\tau_{2}}^{\prime}\right)=\left\langle\nu_{1}, \tau_{1}\right\rangle\left\langle\nu_{1},|y|\right\rangle\left\langle|x|, \tau_{1}\right\rangle \xi\left(\tau_{1}, \nu_{2}\right) \phi(x, y) .
$$

This can be shown as follows.

$$
\begin{aligned}
& \phi\left(K_{\nu_{1}} x J_{\nu_{2}}, K_{\tau_{1}}^{\prime} y J_{\tau_{2}}^{\prime}\right)=\varepsilon_{-}\left(\mathcal{J}_{\nu_{2}}\left({ }_{x} \mathcal{S}\left(\mathcal{K}_{\nu_{1}}\left(K_{\tau_{1}}^{\prime} y J_{\tau_{2}}^{\prime}\right)\right)\right)\right) \\
= & \left\langle\nu_{1}, \tau_{1}\right\rangle\left\langle\nu_{1},|y|\right\rangle\left\langle|x|, \tau_{1}\right\rangle \varepsilon_{-}\left(\mathcal{J}_{\nu_{2}}\left(K_{\tau_{1}}^{\prime} \mathcal{S} \mathcal{S}(y) J_{\tau_{2}}^{\prime}\right)\right) \\
= & \left\langle\nu_{1}, \tau_{1}\right\rangle\left\langle\nu_{1},|y|\right\rangle\left\langle|x|, \tau_{1}\right\rangle \xi\left(\tau_{1}, \nu_{2}\right) \phi(x, y),
\end{aligned}
$$

where ${ }_{x} \mathcal{S}=\rho^{+}(x)$. It's clear $\left|{ }_{x} \mathcal{S}(y)\right|=0$.

Proposition 4. The bilinear form $\phi$ defined in (16) is a skew Hopf pairing.

Proof. Part (a) in Definition 1 follows directly from the definition of $\phi$.

We show (b) in Definition 1. For any $\nu_{1}, \nu_{2}, \mu_{1}, \mu_{2}, \tau_{1}, \tau_{2} \in \mathbb{Z}[I]$ and any $x \in \mathcal{f}^{+}, y^{\prime}, y^{\prime \prime} \in \mathcal{f}^{-}$, by (17), we have

$$
\begin{gathered}
\phi\left(K_{\nu_{1}} x J_{\nu_{2}}, K_{\mu_{1}}^{\prime} y^{\prime} J_{\mu_{2}}^{\prime} K_{\tau_{1}}^{\prime} y^{\prime \prime} J_{\tau_{2}}^{\prime}\right)= \\
\left\langle\left|y^{\prime}\right|, \tau_{1}\right\rangle^{-1} \xi\left(\tau_{1},\left|y^{\prime}\right|\right)\left\langle\nu_{1}+|x|, \mu_{1}+\tau_{1}\right\rangle\left\langle\nu_{1},|x|\right\rangle \xi\left(\mu_{1}+\tau_{1}, \nu_{2}\right) \phi\left(x, y^{\prime} y^{\prime \prime}\right) .
\end{gathered}
$$

By the definition of $\Delta_{+}$, we may write $\Delta_{+}(x)=\sum K_{\left|x_{2}\right|} x_{1} \otimes x_{2} J_{\left|x_{1}\right|}$ with $x_{1}, x_{2} \in^{\prime} \mathfrak{f}^{+}$. By (17), we have

$$
\begin{aligned}
& \phi\left(\Delta_{+}\left(K_{\nu_{1}} x J_{\nu_{2}}\right), K_{\mu_{1}}^{\prime} y^{\prime} J_{\mu_{2}}^{\prime} \otimes K_{\tau_{1}}^{\prime} y^{\prime \prime} J_{\tau_{2}}^{\prime}\right)=\left\langle\left|x_{2}\right|, \tau_{1}\right\rangle \xi\left(\tau_{1},\left|x_{1}\right|\right) \\
& \left\langle|x|, \mu_{1}\right\rangle\left\langle\nu_{1}, \mu_{1}+\tau_{1}+|x|\right\rangle \xi\left(\mu_{1}+\tau_{1}, \nu_{2}\right) \phi\left(\Delta_{+}(x), y^{\prime} \otimes y^{\prime \prime}\right) .
\end{aligned}
$$

By comparing (18) and (19) with $\left|x_{1}\right|=\left|y^{\prime}\right|,\left|x_{2}\right|=\left|y^{\prime \prime}\right|$, (b) is reduced to

$$
\phi\left(x, y^{\prime} y^{\prime \prime}\right)=\phi\left(\Delta_{+}(x), y^{\prime} \otimes y^{\prime \prime}\right),
$$

where $\phi\left(x^{\prime} \otimes x^{\prime \prime}, y^{\prime} \otimes y^{\prime \prime}\right)=\phi\left(x^{\prime}, y^{\prime}\right) \phi\left(x^{\prime \prime}, y^{\prime \prime}\right)$, for all $x, x^{\prime}, x^{\prime \prime} \in{ }^{\prime} \mathfrak{f}^{+}, y^{\prime}, y^{\prime \prime} \in \in^{\prime} \mathfrak{f}^{-}$.

Let us simply write $\Delta_{+}(x)=\sum x_{1} \otimes x_{2}$ with $x_{1}, x_{2} \in \widehat{\mathfrak{f}^{+}}$, not necessarily in $\hat{\mathcal{f}}^{+}$. By the definition of $\phi$, (20) is equivalent to

$$
\rho^{+}(x)\left(y^{\prime} y^{\prime \prime}\right)=\sum \rho^{+}\left(x_{1}\right)\left(y^{\prime}\right) \rho^{+}\left(x_{2}\right)\left(y^{\prime \prime}\right) .
$$


We show (21) by induction on $|x|$. If $x=E_{i}$, (21) can be directly shown by the definition of $\phi$. Assume that (21) holds for $x^{\prime}, x^{\prime \prime}$. We write $\Delta_{+}\left(x^{\prime}\right)=$ $\sum x_{1}^{\prime} \otimes x_{2}^{\prime}$ and $\Delta_{+}\left(x^{\prime \prime}\right)=\sum x_{1}^{\prime \prime} \otimes x_{2}^{\prime \prime}$. Then $\Delta_{+}\left(x^{\prime} x^{\prime \prime}\right)=\sum x_{1}^{\prime} x_{1}^{\prime \prime} \otimes x_{2}^{\prime} x_{2}^{\prime \prime}$ and

$$
\begin{aligned}
\rho^{+}\left(x^{\prime} x^{\prime \prime}\right)\left(y^{\prime} y^{\prime \prime}\right) & =\rho^{+}\left(x^{\prime \prime}\right) \rho^{+}\left(x^{\prime}\right)\left(y^{\prime} y^{\prime \prime}\right) \\
& =\sum \rho^{+}\left(x_{1}^{\prime \prime}\right) \rho^{+}\left(x_{1}^{\prime}\right)\left(y^{\prime}\right) \rho^{+}\left(x_{2}^{\prime \prime}\right) \rho^{+}\left(x_{2}^{\prime}\right)\left(y^{\prime \prime}\right) \\
& =\sum \rho^{+}\left(x_{1}^{\prime} x_{1}^{\prime \prime}\right)\left(y^{\prime}\right) \rho^{+}\left(x_{2}^{\prime} x_{2}^{\prime \prime}\right)\left(y^{\prime \prime}\right) .
\end{aligned}
$$

This proves (21) and, therefore, (b).

We show (c) in Definition 1. By using (17) and a similar argument as we did for (b), (c) is reduced to

$$
\phi\left(x^{\prime} x^{\prime \prime}, y\right)=\phi\left(x^{\prime} \otimes x^{\prime \prime}, \Delta_{-}^{\mathrm{op}}(y)\right), \quad \forall x^{\prime}, x^{\prime \prime} \in^{\prime} \mathfrak{f}^{+}, y \in^{\prime} \mathfrak{f}^{-} .
$$

We show (22) by induction on $|y|$. The case that $y=F_{i}$ is straightforward. Assume that (22) holds for $y^{\prime}, y^{\prime \prime}$ in the second component. We write $\Delta_{-}^{\mathrm{op}}\left(y^{\prime}\right)=\sum y_{1}^{\prime} \otimes y_{2}^{\prime}$ and $\Delta_{-}^{\mathrm{op}}\left(y^{\prime \prime}\right)=\sum y_{1}^{\prime \prime} \otimes y_{2}^{\prime \prime}$. By (20), we have

$$
\begin{aligned}
\phi\left(x^{\prime} x^{\prime \prime}, y^{\prime} y^{\prime \prime}\right) & =\phi\left(\Delta_{+}\left(x^{\prime} x^{\prime \prime}\right), y^{\prime} \otimes y^{\prime \prime}\right)=\sum \phi\left(x_{1}^{\prime} x_{1}^{\prime \prime}, y^{\prime}\right) \phi\left(x_{2}^{\prime} x_{2}^{\prime \prime}, y^{\prime \prime}\right) \\
& =\sum \phi\left(x_{1}^{\prime} \otimes x_{1}^{\prime \prime}, \Delta_{-}^{\mathrm{op}}\left(y^{\prime}\right)\right) \phi\left(x_{2}^{\prime} \otimes x_{2}^{\prime \prime}, \Delta_{-}^{\mathrm{op}}\left(y^{\prime \prime}\right)\right) \\
& =\sum \phi\left(x_{1}^{\prime}, y_{1}^{\prime}\right) \phi\left(x_{2}^{\prime}, y_{1}^{\prime \prime}\right) \phi\left(x_{1}^{\prime \prime}, y_{2}^{\prime}\right) \phi\left(x_{2}^{\prime \prime}, y_{2}^{\prime \prime}\right) \\
& =\sum \phi\left(x^{\prime}, y_{1}^{\prime} y_{1}^{\prime \prime}\right) \phi\left(x^{\prime \prime}, y_{2}^{\prime} y_{2}^{\prime \prime}\right) \\
& =\sum \phi\left(x^{\prime} \otimes x^{\prime \prime}, y_{1}^{\prime} y_{1}^{\prime \prime} \otimes y_{2}^{\prime} y_{2}^{\prime \prime}\right)=\phi\left(x^{\prime} \otimes x^{\prime \prime}, \Delta_{-}^{\mathrm{op}}\left(y^{\prime} y^{\prime \prime}\right)\right) .
\end{aligned}
$$

This proves (c).

We now show (d) in Definition 1. By definition of $S_{+}$, for any $x \in \mathfrak{f}^{+}$, $S_{+}^{\prime}(x)=K_{-|x|} x^{\prime} J_{-|x|}$ with $x^{\prime} \in \mathcal{f}^{+}$and $\left|x^{\prime}\right|=|x|$, where $K_{-|x|}=K_{|x|}^{-1}$ and $J_{-|x|}=J_{|x|}^{-1}$. Since $S_{-}^{-1}\left(F_{i}\right)=-K_{i}^{\prime-1} F_{i} J_{i}^{\prime-1}$, for any $y \in \mathcal{F}^{-}$, we can write $S_{-}^{-1}(y)=K_{-|y|}^{\prime} y^{\prime} J_{-|y|}^{\prime}$ with $y^{\prime} \in \mathcal{f}^{-}$and $\left|y^{\prime}\right|=|y|$. By (17), for any $\nu_{1}, \nu_{2}, \tau_{1}, \tau_{2} \in \mathbb{Z}[I]$ and any $x \in \mathfrak{f}^{+}, y \in \mathcal{f}^{-}$, we have

$$
\begin{aligned}
& \phi\left(S_{+}\left(K_{\nu_{1}} x J_{\nu_{2}}\right), K_{\tau_{1}}^{\prime} y J_{\tau_{2}}^{\prime}\right) \\
& =\left\langle-\nu_{1}, \tau_{1}\right\rangle\left\langle\nu_{1},|x|\right\rangle\left\langle-\nu_{1},|y|\right\rangle \xi\left(|x|, \nu_{2}\right)^{-1} \xi\left(\tau_{1},-\nu_{2}-|x|\right) \phi\left(S_{+}(x), y\right) ; \\
& \phi\left(K_{\nu_{1}} x J_{\nu_{2}}, S_{-}^{\prime-1}\left(K_{\tau_{1}}^{\prime} y J_{\tau_{2}}^{\prime}\right)\right) \\
& =\left\langle|y|, \tau_{1}\right\rangle\left\langle|x|,-\tau_{1}\right\rangle\left\langle\nu_{1},-\tau_{1}\right\rangle \xi\left(-\tau_{1}-|y|, \nu_{2}\right) \xi\left(\tau_{1},|y|\right)^{-1} \phi\left(x, S_{-}^{\prime-1}(y)\right) .
\end{aligned}
$$

By using $|x|=|y|$ and comparing two identities in (23), (d) is reduced to

$$
\phi\left(S_{+}(x), y\right)=\phi\left(x, S_{-}^{-1}(y)\right), \quad \forall x \in \mathfrak{f}^{+}, y \in \mathfrak{f}^{-} .
$$

We show (24) by induction on $|x|$. The case that $x=E_{i}$ is straightforward. Assume that (24) holds for $x^{\prime}, y^{\prime}$ and $x^{\prime \prime}, y^{\prime \prime}$, respectively. We write $\Delta_{-}\left(y^{\prime}\right)=$ $\sum y_{1}^{\prime} \otimes y_{2}^{\prime}$ and $\Delta_{-}\left(y^{\prime \prime}\right)=\sum y_{1}^{\prime \prime} \otimes y_{2}^{\prime \prime}$.

Recall that $\Delta_{-}^{\mathrm{op}} S_{-}^{\prime-1}=\tau_{12}\left(S_{-}^{\prime-1} \otimes S_{-}^{\prime-1}\right) \Delta_{-}^{\mathrm{op}}$, where $\tau_{12}$ is the operator interchanging the first and second component of the tensors. By using this 
identity, we have

$$
\begin{aligned}
\phi\left(S_{+}\left(x^{\prime} x^{\prime \prime}\right), y^{\prime} y^{\prime \prime}\right) & =\phi\left(S_{+}\left(x^{\prime \prime}\right) \otimes S_{+}\left(x^{\prime}\right), \Delta_{-}^{\mathrm{op}}\left(y^{\prime} y^{\prime \prime}\right)\right) \\
& =\sum \phi\left(x^{\prime \prime}, S_{-}^{\prime-1}\left(y_{2}^{\prime} y_{2}^{\prime \prime}\right)\right) \phi\left(x^{\prime}, S_{-}^{\prime-1}\left(y_{1}^{\prime} y_{1}^{\prime \prime}\right)\right) \\
& =\phi\left(x^{\prime} \otimes x^{\prime \prime}, \tau_{12}\left(S_{-}^{\prime-1} \otimes S_{-}^{\prime-1}\right) \Delta_{-}^{\mathrm{op}}\left(y^{\prime} y^{\prime \prime}\right)\right) \\
& =\phi\left(x^{\prime} \otimes x^{\prime \prime}, \Delta_{-}^{\mathrm{op}} S_{-}^{\prime-1}\left(y^{\prime} y^{\prime \prime}\right)\right) \\
& =\phi\left(x^{\prime} x^{\prime \prime}, S_{-}^{-1}\left(y^{\prime} y^{\prime \prime}\right)\right) .
\end{aligned}
$$

This finishes the proof.

Recall $D_{i j}$ from (11), denoted by $\widehat{\mathfrak{J}^{+}}\left(\right.$resp. $\widehat{\mathfrak{J}^{-}}$) the ideal of $\widehat{\mathfrak{f}}^{+}\left(\right.$resp. $\widehat{\mathfrak{f}^{-}}$) generated by $\iota^{+}\left(D_{i j}\right)$ (resp. $\left.\iota^{-}\left(D_{i j}\right)\right)$. Let

$$
\widehat{\mathfrak{f}}^{+}=\widehat{\mathfrak{f}^{+}} / \widehat{\mathfrak{J}^{+}} \text {and } \widehat{\mathfrak{f}}^{-}=\widehat{\prime \mathfrak{f}} / \widehat{\mathfrak{\mathfrak { J }}^{-}} \text {. }
$$

In the rest of this subsection, we shall show that $\phi$ induces a well-defined skew-Hopf pairing $\widehat{\mathfrak{f}}^{+} \times \widehat{\mathfrak{f}}^{-} \rightarrow \mathbb{F}$.

Similarly, for any $\nu, \tau_{1}, \tau_{2} \in \mathbb{Z}[I]$ and any $i, j \in I$, we define $\mathbb{F}$-linear maps $\mathcal{S}_{j}, \mathcal{J}_{\nu}^{\prime}$ and $\mathcal{K}_{\nu}^{\prime}$ from ${ }^{\mathfrak{f}^{+}}$to itself by

$$
\begin{aligned}
& \mathcal{K}_{\nu}^{\prime}\left(K_{\tau_{1}} x J_{\tau_{2}}\right)=\left\langle\tau_{1}, \nu\right\rangle\langle|x|, \nu\rangle \xi(\nu,|x|)^{-1} \xi\left(\nu, \tau_{2}\right) K_{\tau_{1}} x J_{\tau_{2}}, \\
& \mathcal{J}_{\nu}^{\prime}\left(K_{\tau_{1}} x J_{\tau_{2}}\right)=K_{\tau_{1}} x J_{\tau_{2}}, \quad \forall \tau_{1}, \tau_{2} \in \mathbb{Z}[I], x \in^{\prime} \mathfrak{f}^{+}, \\
& \mathcal{S}_{j}\left(K_{\tau_{1}} J_{\tau_{2}}\right)=0, \mathcal{S}_{j}\left(E_{i}\right)=\delta_{i j}, \text { and } \mathcal{S}_{j}(x y)=\mathcal{S}_{j}(x) y+\mathcal{K}_{j}^{\prime}(x) \mathcal{S}_{j}(y) .
\end{aligned}
$$

A direct calculation shows that these maps are well defined.

Let $\mathcal{G}(i)=\xi(i, i)^{1 / 2}, \forall i \in I$. In general, there are two different choices for $\mathcal{G}(i)$, we shall choose one such that

$$
\mathcal{G}\left(\nu_{1}\right) \mathcal{G}\left(\nu_{2}\right) \xi\left(\nu_{2}, \nu_{1}\right)=\mathcal{G}\left(\nu_{1}+\nu_{2}\right), \quad \forall \nu_{1}, \nu_{2} \in \mathbb{N}[I] .
$$

Let $\rho^{-}: \widehat{\mathfrak{f}^{-}} \rightarrow \operatorname{Hom}_{\mathbb{F}}\left(\widehat{\mathfrak{f}^{+}}, \widehat{\mathfrak{f}^{+}}\right)$be the map defined by

$$
F_{j} \mapsto \frac{\mathcal{G}(j)}{v_{j}^{-1}-v_{j}} \mathcal{S}_{j}, \quad K_{\nu}^{\prime} \mapsto \mathcal{K}_{\nu}^{\prime} \quad \text { and } \quad J_{\nu}^{\prime} \mapsto \mathcal{J}_{\nu}^{\prime} .
$$

Then we have the following lemma.

Lemma 5. (i) $\rho^{-}$is an algebra anti-homomorphism.

(ii) For any $y \in^{\prime} \mathfrak{f}^{-}, x^{\prime}, x^{\prime \prime} \in \mathcal{\prime}^{\prime}$, we have

$$
\rho^{-}(y)\left(x^{\prime} x^{\prime \prime}\right)=\sum \rho^{-}\left(y_{2}\right)\left(x^{\prime}\right) \rho^{-}\left(y_{1}\right)\left(x^{\prime \prime}\right),
$$

where $\Delta_{-}(y)=\sum y_{1} \otimes y_{2}$.

The proof is similar to those for Lemma 4 and (21).

We now define a new bilinear form $\phi^{\prime}: \widehat{\widehat{\mathfrak{f}^{+}}} \times \widehat{\mathfrak{f}^{-}} \rightarrow \mathbb{F}$ by

$$
\phi^{\prime}(x, y)=\varepsilon_{+}\left(\rho^{-}(y) x\right), \forall x \in \widehat{\mathfrak{f}^{+}}, y \in \widehat{\mathfrak{f}^{-}} .
$$

The bilinear form $\phi^{\prime}$ is well defined. 
By Lemma 5(ii), we have

$$
\phi^{\prime}\left(x^{\prime} x^{\prime \prime}, y\right)=\phi^{\prime}\left(x^{\prime} \otimes x^{\prime \prime}, \Delta_{-}^{\mathrm{op}}(y)\right)
$$

where $\phi^{\prime}\left(x^{\prime} \otimes x^{\prime \prime}, y^{\prime} \otimes y^{\prime \prime}\right)=\phi^{\prime}\left(x^{\prime}, y^{\prime}\right) \phi^{\prime}\left(x^{\prime \prime}, y^{\prime \prime}\right)$, for all $x^{\prime}, x^{\prime \prime} \in^{\prime} \mathfrak{f}^{+}, y, y^{\prime}, y^{\prime \prime} \in$ $\mathfrak{f}^{-}$.

Moreover, for any $\nu_{1}, \nu_{2}, \tau_{1}, \tau_{2} \in \mathbb{Z}[I]$ and $x \in \mathfrak{f}^{\prime}, y \in \mathfrak{\prime}^{-}$, we have

$$
\begin{gathered}
\phi^{\prime}\left(K_{\nu_{1}} x J_{\nu_{2}}, K_{\tau_{1}}^{\prime} y J_{\tau_{2}}^{\prime}\right)= \\
\left\langle\nu_{1}, \tau_{1}\right\rangle\left\langle\nu_{1},|y|\right\rangle\left\langle|x|, \tau_{1}\right\rangle \xi\left(\tau_{1},|x|\right)^{-1} \xi\left(\tau_{1}, \nu_{2}\right) \phi^{\prime}(x, y) .
\end{gathered}
$$

This equality can be directly checked by the definition of $\phi^{\prime}$. We notice that the coefficient on the right hand side of (27) is different from the one in (17). The difference is $\xi\left(\tau_{1},|x|\right)^{-1}$.

Lemma 6. For any homogenous elements $x \in \mathfrak{1}^{+}$and $y \in \mathfrak{f}^{-}$, we have $\phi(x, y)=\mathcal{G}(|x|) \phi^{\prime}(x, y)$.

Proof. We show it by induction on $|x|$. If $|x|=i$, we have

$$
\phi\left(E_{i}, F_{j}\right)=\delta_{i j} \frac{\xi(i, i)}{v_{i}^{-1}-v_{i}}=\mathcal{G}(i) \phi^{\prime}\left(E_{i}, F_{j}\right) .
$$

Assume that the lemma holds for $x^{\prime}, x^{\prime \prime} \in \mathfrak{f}^{+}$in the first component. For any $y \in \in^{\prime} \mathfrak{f}^{-}$, we write $\Delta_{-}^{\mathrm{op}}(y)=\sum K_{\left|y_{1}\right|}^{\prime} y_{2} \otimes y_{1} J_{\left|y_{2}\right|}^{\prime}$ with $y_{1}, y_{2} \in \in^{\prime} \mathfrak{f}^{-}$. Then we have

$$
\begin{aligned}
& \phi\left(x^{\prime} x^{\prime \prime}, y\right)=\sum \phi\left(x^{\prime}, K_{\left|y_{1}\right|}^{\prime} y_{2}\right) \phi\left(x^{\prime \prime}, y_{1} J_{\left|y_{2}\right|}^{\prime}\right) \\
= & \sum\left\langle\left|x^{\prime}\right|,\left|y_{1}\right|\right\rangle \mathcal{G}\left(\left|x^{\prime}\right|\right) \mathcal{G}\left(\left|x^{\prime \prime}\right|\right) \phi^{\prime}\left(x^{\prime}, y_{2}\right) \phi^{\prime}\left(x^{\prime \prime}, y_{1}\right) \\
= & \sum \mathcal{G}\left(\left|x^{\prime}\right|\right) \mathcal{G}\left(\left|x^{\prime \prime}\right|\right) \xi\left(\left|y_{1}\right|,\left|x^{\prime}\right|\right) \phi^{\prime}\left(x^{\prime}, K_{\left|y_{1}\right|}^{\prime} y_{2}\right) \phi^{\prime}\left(x^{\prime \prime}, y_{1} J_{\left|y_{2}\right|}^{\prime}\right) \\
= & \mathcal{G}\left(\left|x^{\prime}\right|+\left|x^{\prime \prime}\right|\right) \phi^{\prime}\left(x^{\prime} x^{\prime \prime}, y\right) .
\end{aligned}
$$

Here we use the fact that $\left|x^{\prime \prime}\right|=\left|y_{1}\right|$. This finishes the proof.

By Proposition 2, we have

$$
{ }_{l} r\left(D_{i j}\right)=0=r_{l}\left(D_{i j}\right), \quad \forall i \neq j, l \in I .
$$

By comparing the definition of ${ }_{l} r$ with that of $\mathcal{S}_{l}$ in (25), we have

$$
\left.\mathcal{S}_{l}\right|_{\mathfrak{f}^{+}} \circ \iota^{+}=\iota^{+} \circ{ }_{l} r
$$

Lemma 7. For any homogenous elements $x \in \in^{\prime} \mathfrak{f}$ and $i \in I$, we have

$$
\left.{ }_{i} \mathcal{S}\right|_{\mathfrak{f}^{-}} \circ \iota^{-}(x)=\xi\left(\left|x^{-}\right|, i\right) \iota^{-} \circ r_{i}(x),
$$

where $x^{-}=\iota^{-}(x)$.

Proof. We show it by induction on $\left|x^{-}\right|$. Recall the definition of ${ }_{i} \mathcal{S}$ from (15), the case that $\left|x^{-}\right|=j$ is trivial. Assume that the lemma holds for $x, y$. 
Then we have

$$
\begin{aligned}
& \left.i \mathcal{S}\right|_{\mathfrak{f}^{-}} \circ \iota^{-}(x y)=\left.\xi\left(\left|x^{-}\right|, i\right)_{i} \mathcal{S}\right|_{\mathfrak{f}^{-}}\left(y^{-}\right) x^{-}+\left.\left\langle i,\left|y^{-}\right|\right\rangle y^{-}{ }_{i} \mathcal{S}\right|_{\mathfrak{f}^{-}}\left(x^{-}\right) \\
= & \xi\left(\left|x^{-}\right|+\left|y^{-}\right|, i\right) \iota^{-} \circ r_{i}(y) x^{-}+\left\langle i,\left|y^{-}\right|\right\rangle \xi\left(\left|x^{-}\right|, i\right) y^{-} \iota^{-} \circ r_{i}(x) \\
= & \xi\left(\left|x^{-}\right|+\left|y^{-}\right|, i\right)\left(\iota^{-} \circ r_{i}(y) x^{-}+\left\langle i,\left|y^{-}\right|\right\rangle \xi\left(\left|y^{-}\right|, i\right)^{-1} y^{-} \iota^{-} \circ r_{i}(x)\right) \\
= & \xi\left(\left|x^{-}\right|+\left|y^{-}\right|, i\right) \iota^{-} \circ r_{i}(x y) .
\end{aligned}
$$

Lemma is proved.

By (28), (29) and Lemma 7, we have

$$
\begin{aligned}
& \mathcal{S}_{l}\left(\iota^{+}\left(D_{i j}\right)\right)=\iota^{+} \circ{ }_{l} r\left(D_{i j}\right)=0, \\
& { }_{l} \mathcal{S}\left(\iota^{-}\left(D_{i j}\right)\right)=\xi\left(\left|D_{i j}^{-}\right|, l\right) \iota^{-} \circ r_{l}\left(D_{i j}\right)=0, \quad \forall i \neq j, l \in I .
\end{aligned}
$$

Corollary 1. The bilinear form $\phi$ induces a well defined skew-Hopf pairing $\widehat{\mathfrak{f}}^{+} \times \widehat{\mathfrak{f}}^{-} \rightarrow \mathbb{F}$.

Proof. By the definition of $\widehat{\mathfrak{f}}^{+}$and $\widehat{\mathfrak{f}}^{-}$, it is enough to show that $\widehat{\mathfrak{J}^{+}}$and $\widehat{\mathfrak{J}^{-}}$ are in the radical of $\phi$, i.e., $\phi\left(\iota^{+}\left(D_{i j}\right), y\right)=0=\phi\left(x, \iota^{-}\left(D_{i j}\right)\right)$ for any $i \neq j$, $x \in \widehat{\hat{\mathfrak{f}}}^{+}$and $y \in \widehat{\widehat{\mathfrak{f}}}^{-}$. By (17), we may assume that $x \in \mathfrak{\mathcal { F }}^{+}$and $y \in \mathfrak{\mathcal { F }}^{-}$. If $|x|=|y|=0$, there is nothing to show. We now assume that $|x|$ and $|y|$ are not equal to zero. Then we can write $x=E_{l} x^{\prime}$ for some $l$. By the definition of $\phi$ and (30), we have

$$
\phi\left(x, \iota^{-}\left(D_{i j}\right)\right)=\varepsilon_{-}\left(\rho^{+}\left(x^{\prime}\right)_{l} \mathcal{S}\left(\iota^{-}\left(D_{i j}\right)\right)\right)=0, \quad \forall i \neq j .
$$

Similarly, $\phi^{\prime}\left(\iota^{+}\left(D_{i j}, y\right)\right)=0$ for any $i \neq j$. By Lemma 6, we have

$$
\phi\left(\iota^{+}\left(D_{i j}\right), y\right)=\mathcal{G}\left(\left|D_{i j}\right|\right) \phi^{\prime}\left(\iota^{+}\left(D_{i j}\right), y\right)=0, \quad \forall i \neq j .
$$

This finishes the proof.

By Proposition 3, there is a Hopf algebra structure on $\widehat{\mathfrak{f}}^{+} \otimes \widehat{\mathfrak{f}}^{-}$, denoted this Hopf algebra by $\mathbf{U}_{\beta, \xi}$.

3.4. Algebraic presentation of $\mathbf{U}_{\beta, \xi}$. It is clear that $\mathbf{U}_{\beta, \xi}$ is generated by $E_{i}, F_{i}, J_{i}^{ \pm 1}, J_{i}^{\prime \pm 1}, K_{i}^{ \pm 1}$ and $K_{i}^{\prime \pm 1}$ for all $i \in I$. We now calculate defining relations of $\mathbf{U}_{\beta, \xi}$ in these generators.

Since $\widehat{\mathfrak{f}}^{+}$and $\widehat{\mathfrak{f}}^{-}$can be thought as subalgebras of $\mathbf{U}_{\beta, \xi}$, the algebra $\mathbf{U}_{\beta, \xi}$ carries on all defining relations of $\widehat{\mathfrak{f}}^{+}$and $\widehat{\mathfrak{f}}^{-}$. It is easy to show that $J_{i}^{ \pm 1}, J_{i}^{\prime \pm 1}, K_{i}^{ \pm 1}$ and $K_{i}^{\prime \pm 1}$ commute with each other.

Since $\Delta_{-}^{2}\left(J_{i}^{\prime}\right)=J_{i}^{\prime} \otimes J_{i}^{\prime} \otimes J_{i}^{\prime}, \Delta_{-}^{2}\left(K_{i}^{\prime}\right)=K_{i}^{\prime} \otimes K_{i}^{\prime} \otimes K_{i}^{\prime}$ and

$$
\Delta_{+}^{2}\left(E_{i}\right)=E_{i} \otimes J_{i} \otimes J_{i}+K_{i} \otimes E_{i} \otimes J_{i}+K_{i} \otimes K_{i} \otimes E_{i},
$$

by (iii) in Proposition 3 , we have

$$
\begin{gathered}
K_{i}^{\prime} E_{j}=\phi\left(K_{j}, K_{i}^{\prime-1}\right) E_{j} K_{i}^{\prime} \phi\left(J_{j}, K_{i}^{\prime}\right)=\langle j, i\rangle^{-1} \xi(i, j) E_{j} K_{i}^{\prime}, \text { and } \\
J_{i}^{\prime} E_{j}=\phi\left(K_{j}, J_{i}^{\prime-1}\right) E_{j} J_{i}^{\prime} \phi\left(J_{j}, J_{i}^{\prime}\right)=E_{j} J_{i}^{\prime} .
\end{gathered}
$$


Since $\Delta_{+}^{2}\left(J_{i}\right)=J_{i} \otimes J_{i} \otimes J_{i}, \Delta_{+}^{2}\left(K_{i}\right)=K_{i} \otimes K_{i} \otimes K_{i}$ and

$$
\Delta_{-}^{2}\left(F_{i}\right)=J_{i}^{\prime} \otimes J_{i}^{\prime} \otimes F_{i}+J_{i}^{\prime} \otimes F_{i} \otimes K_{i}^{\prime}+F_{i} \otimes K_{i}^{\prime} \otimes K_{i}^{\prime},
$$

we have

$$
\begin{gathered}
F_{j} K_{i}=\phi\left(K_{i}, J_{j}^{\prime-1}\right) K_{i} F_{j} \phi\left(K_{i}, K_{j}^{\prime}\right)=\langle i, j\rangle K_{i} F_{j}, \text { and } \\
F_{j} J_{i}=\phi\left(J_{i}, J_{i}^{\prime-1}\right) J_{i} F_{j} \phi\left(J_{i}, K_{j}^{\prime}\right)=\xi(j, i) J_{i} F_{j} .
\end{gathered}
$$

Similarly, by (31) and (32), we have

$$
\begin{gathered}
F_{j} E_{i}=\phi\left(E_{i},-J_{j}^{\prime-1} F_{j} K_{j}^{\prime-1}\right) J_{i} K_{j}^{\prime} \phi\left(J_{i}, K_{j}^{\prime}\right)+E_{i} F_{j} \phi\left(J_{i}, K_{j}^{\prime}\right)+K_{i} J_{j}^{\prime} \phi\left(E_{i}, F_{j}\right) \\
=\xi(j, i) E_{i} F_{j}+\delta_{i j} \frac{\xi(j, i)}{v_{i}^{-1}-v_{i}}\left(K_{i} J_{j}^{\prime}-J_{i} K_{j}^{\prime}\right) .
\end{gathered}
$$

Summarizing up, we have the following presentation of $\mathbf{U}_{\beta, \xi}$ generated by symbols $E_{i}, F_{i}, J_{i}^{ \pm 1}, J_{i}^{\prime \pm 1}, K_{i}^{ \pm 1}, K_{i}^{\prime \pm 1}, \forall i \in I$, and subjects to the following relations.

(R1) $\quad J_{i}^{ \pm 1}, J_{i}^{\prime \pm 1}, K_{i}^{ \pm 1}$ and $K_{i}^{ \pm 1}$ commute with each other,

$$
\begin{aligned}
& K_{i}^{ \pm 1} K_{i}^{\mp 1}=K_{i}^{\prime \pm 1} K_{i}^{\prime \mp 1}=J_{i}^{ \pm 1} J_{i}^{\mp 1}=J_{i}^{\prime \pm 1} J_{i}^{\prime \mp 1}=1 . \\
& K_{i} E_{j}=\langle i, j\rangle E_{j} K_{i}, \quad K_{i}^{\prime} E_{j}=\langle j, i\rangle^{-1} \xi(i, j) E_{j} K_{i}^{\prime}, \\
& K_{i} F_{j}=\langle i, j\rangle^{-1} F_{j} K_{i}, \quad K_{i}^{\prime} F_{j}=\langle j, i\rangle \xi(i, j)^{-1} F_{j} K_{i}^{\prime}, \\
& J_{i} E_{j}=\xi(j, i) E_{j} J_{i}, \quad J_{i}^{\prime} E_{j}=E_{j} J_{i}^{\prime}, \\
& J_{i} F_{j}=\xi(j, i)^{-1} F_{j} J_{i}, \quad J_{i}^{\prime} F_{j}=F_{j} J_{i}^{\prime} . \\
& E_{i} F_{j}-\xi(j, i)^{-1} F_{j} E_{i}=\delta_{i j} \frac{K_{i} J_{i}^{\prime}-J_{i} K_{i}^{\prime}}{v_{i}-v_{i}^{-1}} . \\
& \quad \sum_{k+k^{\prime}=1-a_{i j}}(-1)^{k}(\beta(i, j))^{-k} E_{i}^{(k)} E_{j} E_{i}^{\left(k^{\prime}\right)}=0, \quad \text { if } i \neq j, \\
& \quad \sum_{k+k^{\prime}=1-a_{i j}}(-1)^{k}(\beta(i, j))^{-k} F_{i}^{\left(k^{\prime}\right)} F_{j} F_{i}^{(k)}=0, \quad \text { if } i \neq j .
\end{aligned}
$$

We note that $\mathbf{U}_{\beta, \xi}$ admits a triangle decomposition $\mathbf{U}_{\beta, \xi} \simeq \mathbf{U}^{+} \otimes \mathbf{U}^{0} \otimes \mathbf{U}^{-}$ by repeating the argument for ordinary quantum groups word by word, where $\mathbf{U}^{+}$(resp. $\mathbf{U}^{0}$ or $\mathbf{U}^{-}$) is a subalgebra of $\mathbf{U}_{\beta, \xi}$ generated by $E_{i}$ (resp. $J_{i}^{ \pm 1}, J_{i}^{\prime \pm 1}, K_{i}^{ \pm 1}, K_{i}^{ \pm 1}$ or $F_{i}$ ) for all $i \in I$.

3.5. Hopf algebra structure on $\mathbf{U}_{\beta, \xi}$ • By Proposition 3 , $\mathbf{U}_{\beta, \xi}$ has a Hopf algebra structure with the comultiplication $\Delta$, the counit $\varepsilon$ and the antipode $S$ given as follows.

$$
\begin{array}{cl}
\Delta\left(E_{i}\right)=E_{i} \otimes J_{i}+K_{i} \otimes E_{i}, & \Delta\left(K_{i}^{ \pm 1}\right)=K_{i}^{ \pm 1} \otimes K_{i}^{ \pm 1}, \\
\Delta\left(F_{i}\right)=J_{i}^{\prime} \otimes F_{i}+F_{i} \otimes K_{i}^{\prime}, & \Delta\left(K_{i}^{ \pm 1}\right)=K_{i}^{ \pm 1} \otimes K_{i}^{\prime \pm 1}, \\
\Delta\left(J_{i}^{ \pm 1}\right)=J_{i}^{ \pm 1} \otimes J_{i}^{ \pm 1}, & \Delta\left(J_{i}^{\prime \pm 1}\right)=J_{i}^{\prime \pm 1} \otimes J_{i}^{\prime \pm 1}, \\
\varepsilon\left(K_{i}^{ \pm 1}\right)=\varepsilon\left(J_{i}^{ \pm 1}\right)=1, & \varepsilon\left(E_{i}\right)=0,
\end{array}
$$




$$
\begin{aligned}
& \varepsilon\left(K_{i}^{\prime \pm 1}\right)=\varepsilon\left(J_{i}^{\prime \pm 1}\right)=1, \quad \varepsilon\left(F_{i}\right)=0, \\
& S\left(E_{i}\right)=-K_{i}^{-1} E_{i} J_{i}^{-1}, \quad S\left(K_{i}^{ \pm 1}\right)=K_{i}^{\mp 1}, \quad S\left(J_{i}^{ \pm 1}\right)=J_{i}^{\mp 1}, \\
& S\left(F_{i}\right)=-J_{i}^{\prime-1} F_{i} K_{i}^{\prime-1}, \quad S\left(K_{i}^{\prime \pm 1}\right)=K_{i}^{\prime \mp 1}, \quad S\left(J_{i}^{\prime \pm 1}\right)=J_{i}^{\prime \mp 1} .
\end{aligned}
$$

\section{Specializations of $\mathbf{U}_{\beta, \xi}$}

In this section, we shall show that various quantum algebras in Section 2.3 admit a Drinfeld double construction by specializing the parameters $\beta(i, j)$ and $\xi(i, j)$, for all $i, j \in I$.

4.1. The entire two-parameter quantum algebras. In the case of the two-parameter quantum algebra (see Example 1), we set

$$
\beta(i, j)=t^{\Omega_{j i}-\Omega_{i j}} \quad \text { and } \quad \xi(i, j)=1, \quad \forall i, j \in I .
$$

Let $\mathcal{G}(\nu)=1$ for all $\nu \in \mathbb{N}[I]$, then (26) holds.

Under these settings, we get the following presentation of an entire twoparameter quantum algebra $\mathbf{U}_{v, t}$, generated by symbols $E_{i}, F_{i}, K_{i}^{ \pm 1}, K_{i}^{\prime \pm 1}$, $J_{i}^{ \pm 1}, J_{i}^{\prime \pm 1}, \forall i \in I$, and subjects to the following relations.

$\left(R_{1} 1\right) \quad J_{i}^{ \pm 1}, J_{i}^{\prime \pm 1}, K_{i}^{ \pm 1}$ and $K_{i}^{\prime \pm 1}$ commute with each other,

$$
K_{i}^{ \pm 1} K_{i}^{\mp 1}=K_{i}^{\prime \pm 1} K_{i}^{\prime \mp 1}=J_{i}^{ \pm 1} J_{i}^{\mp 1}=J_{i}^{\prime \pm 1} J_{i}^{\prime \mp 1}=1 .
$$

$$
\begin{aligned}
& K_{i} E_{j} K_{i}^{-1}=v^{-i \cdot j} t^{\Omega_{j i}-\Omega_{i j}} E_{j}, \quad K_{i}^{\prime} E_{j} K_{i}^{\prime-1}=v^{i \cdot j} t^{\Omega_{j i}-\Omega_{i j}} E_{j}, \\
& K_{i}^{\prime} F_{j} K_{i}^{\prime-1}=v^{-i \cdot j} t^{\Omega_{i j}-\Omega_{j i}} F_{j}, \quad K_{i} F_{j} K_{i}^{-1}=v^{i \cdot j} t^{\Omega_{i j}-\Omega_{j i}} F_{j}, \\
& J_{i} E_{j} J_{i}^{-1}=E_{j}, \quad J_{i}^{\prime} E_{j} J_{i}^{\prime-1}=E_{j}, \quad J_{i} F_{j} J_{i}^{-1}=F_{j}, \quad J_{i}^{\prime} F_{j} J_{i}^{\prime-1}=F_{j} . \\
& E_{i} F_{j}-F_{j} E_{i}=\delta_{i j} \frac{K_{i} J_{i}^{\prime}-J_{i} K_{i}^{\prime}}{v_{i}-v_{i}^{-1}} . \\
& \quad \sum_{k+k^{\prime}=1-a_{i j}}(-1)^{k}\left[\begin{array}{c}
1-a_{i j} \\
k
\end{array}\right]_{v_{i}} t^{k\left(\Omega_{i j}-\Omega_{j i}\right)} E_{i}^{k} E_{j} E_{i}^{k^{\prime}}=0, \quad \text { if } i \neq j, \\
& \sum_{k+k^{\prime}=1-a_{i j}}(-1)^{k}\left[\begin{array}{c}
1-a_{i j} \\
k
\end{array}\right]_{v_{i}} t^{k\left(\Omega_{i j}-\Omega_{j i}\right)} F_{i}^{k^{\prime}} F_{j} F_{i}^{k}=0, \quad \text { if } i \neq j .
\end{aligned}
$$

Let $U_{v, t}$ be the two-parameter quantum algebra in [FL] associated to $(I, \cdot)$.

Proposition 5. The map $\Phi: U_{v, t} \rightarrow \mathbf{U}_{v, t} /\left\langle J_{i}-1, J_{i}^{\prime}-1\right\rangle$ sending $E_{i} \mapsto F_{i}$, $F_{i} \mapsto E_{i}, K_{i} \mapsto-K_{i}$ and $K_{i}^{\prime} \mapsto-K_{i}^{\prime}$ for all $i \in I$ is a $\mathbb{Q}(v, t)$-algebra isomorphism.

By Proposition 5, if we set $J_{i}=J_{i}^{\prime}=1$ in Section 3, then the construction in Section 3 provides a Drinfeld double construction of $U_{v, t}$. This recovers the results in $[\mathrm{BGH}, \mathrm{BW}$. 
4.2. The entire quantum superalgebras. In the case of the quantum superalgebra (see Example 2), we set

$$
\xi(i, j)=t^{2 \mathcal{P}(i) \mathcal{P}(j)} \quad \text { and } \quad \beta(i, j)=t^{i \cdot j} t^{2 \mathcal{P}(i) \mathcal{P}(j)}, \forall i, j \in I,
$$

where $t=\mathbf{i}$.

Then, $\langle i, j\rangle=\left(v^{-1} t\right)^{i \cdot j}$. We have

$$
\xi(i, j) \xi(j, i)=1 \quad \text { and } \quad\langle i, j\rangle=\langle j, i\rangle, \forall i, j \in I .
$$

Let $\mathcal{G}(\nu)=t^{(\mathcal{P}(\nu))^{2}}$ for all $\nu \in \mathbb{N}[I]$, where $\mathcal{P}(\nu)=\sum_{i} \nu_{i} \mathcal{P}(i)$. It's easy to check that $\mathcal{G}(\nu)$ satisfies (26). Under these settings, we have the following presentation of an entire quantum superalgebra $\mathbf{U}_{v, \mathbf{i}}$ generated by symbols $E_{i}, F_{i}, J_{i}^{ \pm 1}, J_{i}^{\prime \pm 1}, K_{i}^{ \pm 1}, K_{i}^{\prime \pm 1}, \forall i \in I$, and subjects to the following relations.

$\left(R_{2} 1\right) \quad J_{i}^{ \pm 1}, J_{i}^{\prime \pm 1}, K_{i}^{ \pm 1}$ and $K_{i}^{\prime \pm 1}$ commute with each other, $K_{i}^{ \pm 1} K_{i}^{\mp 1}=K_{i}^{\prime \pm 1} K_{i}^{\prime \mp 1}=J_{i}^{ \pm 1} J_{i}^{\mp 1}=J_{i}^{\prime \pm 1} J_{i}^{\prime \mp 1}=1$.

$$
\begin{aligned}
& K_{i} E_{j}=v^{-i \cdot j} t^{i \cdot j} E_{j} K_{i}, \quad K_{i}^{\prime} E_{j}=v^{i \cdot j} t^{-i \cdot j} t^{2 \mathcal{P}(i) \mathcal{P}(j)} E_{j} K_{i}^{\prime}, \\
& K_{i} F_{j}=v^{i \cdot j} t^{-i \cdot j} F_{j} K_{i}, \quad K_{i}^{\prime} F_{j}=v^{-i \cdot j} t^{i \cdot j} t^{2 \mathcal{P}(i) \mathcal{P}(j)} F_{j} K_{i}^{\prime}, \\
& J_{i} E_{j}=t^{2 \mathcal{P}(i) \mathcal{P}(j)} E_{j} J_{i}, \quad J_{i}^{\prime} E_{j}=E_{j} J_{i}^{\prime}, \\
& J_{i} F_{j}=t^{2 \mathcal{P}(i) \mathcal{P}(j)} F_{j} J_{i}, \quad J_{i}^{\prime} F_{j}=F_{j} J_{i}^{\prime} .
\end{aligned}
$$

$$
E_{i} F_{j}-t^{2 \mathcal{P}(i) \mathcal{P}(j)} F_{j} E_{i}=\delta_{i j} \frac{K_{i} J_{i}^{\prime}-J_{i} K_{i}^{\prime}}{v_{i}-v_{i}^{-1}}
$$

$$
\begin{gathered}
\sum_{k+k^{\prime}=1-a_{i j}}(-1)^{k}\left[\begin{array}{c}
1-a_{i j} \\
k
\end{array}\right]_{v_{i}} t^{k i \cdot j+2 k \mathcal{P}(i) \mathcal{P}(j)} E_{i}^{k} E_{j} E_{i}^{k^{\prime}}=0, \quad \text { if } i \neq j, \\
\sum_{k+k^{\prime}=1-a_{i j}}(-1)^{k}\left[\begin{array}{c}
1-a_{i j} \\
k
\end{array}\right]_{v_{i}} t^{k i \cdot j+2 k \mathcal{P}(i) \mathcal{P}(j)} F_{i}^{k^{\prime}} F_{j} F_{i}^{k}=0, \quad \text { if } i \neq j .
\end{gathered}
$$

Let $U_{q, \mathbf{i}}$ be the quantum superalgebra defined in [CHW] associated to $(I, \cdot)$ which is a bar-consistent super Cartan datum. Let $\mathcal{I}$ be the ideal of $\mathbf{U}_{v, \mathbf{i}}$ generated by $\left\{K_{i} K_{i}^{\prime} J_{i}-1, \forall i \in I\right\}$, and $\mathbf{U}_{v, \mathbf{i}}^{s q}$ the subalgebra of $\mathbf{U}_{v, \mathbf{i}} / \mathcal{I}$ generated by $E_{i}, F_{i}, J_{i}^{\prime \pm}, K_{i}^{ \pm}$and $\left(J_{i} K_{i}^{\prime}\right)^{ \pm}$. Then we have the following proposition.

Proposition 6. The map $\varsigma: U_{q, \mathbf{i}} \rightarrow \mathbf{U}_{v, \mathbf{i}}^{s q}$ sending

$$
q \mapsto v^{-1} t, E_{i} \mapsto E_{i}, F_{i} \mapsto F_{i}, J_{i} \mapsto t_{i}^{2} J_{i}^{\prime}, K_{i} \mapsto t_{i}^{-1} K_{i}, \quad \forall i \in I
$$

is a $\mathbb{Q}$-algebra isomorphism.

4.3. The entire Multi-parameter quantum algebras. In the case of the multi-parameter quantum algebra (see Example 3), we set

$$
\beta(i, j)=v^{i \cdot j} q_{i j} \quad \text { and } \quad \xi(i, j)=1, \quad \forall i, j \in I .
$$

Let $\mathcal{G}(\nu)=1$ for all $\nu \in \mathbb{N}[I]$, then (26) holds. 
Under these settings, we get the following presentation of an entire multiparameter quantum algebra $\mathbf{U}_{v, \mathbf{q}}$, generated by symbols $E_{i}, F_{i}, K_{i}^{ \pm 1}, K_{i}^{\prime \pm 1}$, $J_{i}^{ \pm 1}, J_{i}^{\prime \pm 1}, \forall i \in I$, and subjects to the following relations.

$\left(R_{3} 1\right) \quad J_{i}^{ \pm 1}, J_{i}^{\prime \pm 1}, K_{i}^{ \pm 1}$ and $K_{i}^{\prime \pm 1}$ commute with each other, $K_{i}^{ \pm 1} K_{i}^{\mp 1}=K_{i}^{\prime \pm 1} K_{i}^{\prime \mp 1}=J_{i}^{ \pm 1} J_{i}^{\mp 1}=J_{i}^{\prime \pm 1} J_{i}^{\prime \mp 1}=1$.

$$
\begin{aligned}
& K_{i} E_{j} K_{i}^{-1}=q_{i j} E_{j}, \quad K_{i}^{\prime} E_{j} K_{i}^{\prime-1}=q_{j i}^{-1} E_{j}, \\
& K_{i}^{\prime} F_{j} K_{i}^{\prime-1}=q_{j i} F_{j}, \quad K_{i} F_{j} K_{i}^{-1}=q_{i j}^{-1} F_{j}, \\
& J_{i} E_{j} J_{i}^{-1}=E_{j}, \quad J_{i}^{\prime} E_{j} J_{i}^{\prime-1}=E_{j}, \quad J_{i} F_{j} J_{i}^{-1}=F_{j}, \quad J_{i}^{\prime} F_{j} J_{i}^{\prime-1}=F_{j} .
\end{aligned}
$$

$$
\begin{aligned}
& E_{i} F_{j}-F_{j} E_{i}=\delta_{i j} \frac{K_{i} J_{i}^{\prime}-J_{i} K_{i}^{\prime}}{v_{i}-v_{i}^{-1}} . \\
& \sum_{k+k^{\prime}=1-a_{i j}}(-1)^{k}\left[\begin{array}{c}
1-a_{i j} \\
k
\end{array}\right]_{v_{i}}\left(v^{i \cdot j} q_{i j}\right)^{-k} E_{i}^{k} E_{j} E_{i}^{k^{\prime}}=0, \quad \text { if } i \neq j, \\
& \sum_{k+k^{\prime}=1-a_{i j}}(-1)^{k}\left[\begin{array}{c}
1-a_{i j} \\
k
\end{array}\right]_{v_{i}}\left(v^{i \cdot j} q_{i j}\right)^{-k} F_{i}^{k^{\prime}} F_{j} F_{i}^{k}=0, \quad \text { if } i \neq j .
\end{aligned}
$$

Let $U_{\mathbf{q}}$ be the multi-parameter quantum algebra in [HPR] associated to $(I, \cdot)$.

Proposition 7. Under the assumption (7), the map $\Psi: U_{\mathbf{q}} \rightarrow \mathbf{U}_{v, \mathbf{q}} /\left\langle J_{i}-\right.$ $\left.1, J_{i}^{\prime}-1\right\rangle$ sending $e_{i} \mapsto E_{i}, f_{i} \mapsto F_{i}, \omega_{i} \mapsto-v_{i} K_{i}$ and $\omega_{i}^{\prime} \mapsto-v_{i} K_{i}^{\prime}$, for all $i \in I$ is a $\mathbb{Q}$-algebra isomorphism.

By Proposition 5, if we set $J_{i}=J_{i}^{\prime}=1$ in Section 3, then the construction in Section 3 provides a Drinfeld double construction of $U_{\mathbf{q}}$. This recovers the result in $\mathrm{HPR}$.

4.4. The entire Multi-parameter quantum superalgebras I. In the case of the multi-parameter quantum super algebra (see Example 4), we further assume that $s_{i j}=s_{j i}$. Then, we set

$$
\xi(i, j)=s_{i j}^{-1} \quad \text { and } \quad \beta(i, j)=s_{j i}^{-1} p_{j i} v_{i}^{-a_{i j}}, \forall i, j \in I .
$$

Let $\mathcal{G}(\nu)=\prod_{i, j \in I} s_{i j}^{-\nu(i) \nu(j) / 2}$, for all $\nu \in \mathbb{N}[I]$ (i.e. $\left.\nu=\sum_{i} \nu(i) i\right)$, then (26) holds. Under these settings, we obtain an entire multi-parameter quantum superalgebra $\mathbf{U}_{\mathbf{s}, \mathbf{p}}$, generated by symbols $E_{i}, F_{i}, K_{i}^{ \pm 1}, K_{i}^{\prime \pm 1}, J_{i}^{ \pm 1}, J_{i}^{\prime \pm 1}, \forall i \in$ $I$, and subjects to the following relations.

$\left(R_{4} 1\right) \quad J_{i}^{ \pm 1}, J_{i}^{\prime \pm 1}, K_{i}^{ \pm 1}$ and $K_{i}^{\prime \pm 1}$ commute with each other, $K_{i}^{ \pm 1} K_{i}^{\mp 1}=K_{i}^{\prime \pm 1} K_{i}^{\prime \mp 1}=J_{i}^{ \pm 1} J_{i}^{\mp 1}=J_{i}^{\prime \pm 1} J_{i}^{\prime \mp 1}=1$.

$\left(R_{4} 2\right) \quad K_{i} E_{j}=p_{i j}^{-1} E_{j} K_{i}, \quad K_{i}^{\prime} E_{j}=p_{j i} s_{j i}^{-1} E_{j} K_{i}^{\prime}$,

$K_{i} F_{j}=p_{i j} F_{j} K_{i}, \quad K_{i}^{\prime} F_{j}=p_{j i}^{-1} s_{j i} F_{j} K_{i}^{\prime}$,

$J_{i} E_{j}=s_{j i}^{-1} E_{j} J_{i}, \quad J_{i}^{\prime} E_{j}=E_{j} J_{i}^{\prime}$, 


$$
\begin{aligned}
& J_{i} F_{j}=s_{j i} F_{j} J_{i}, \quad J_{i}^{\prime} F_{j}=F_{j} J_{i}^{\prime} . \\
& E_{i} F_{j}-s_{j i} F_{j} E_{i}=\delta_{i j} \frac{K_{i} J_{i}^{\prime}-J_{i} K_{i}^{\prime}}{v_{i}-v_{i}^{-1}} . \\
& \sum_{k+k^{\prime}=1-a_{i j}}(-1)^{k}\left[\begin{array}{c}
1-a_{i j} \\
k
\end{array}\right]_{v_{i}} s_{j i}^{k} p_{j i}^{-k} v_{i}^{k a_{i j}} E_{i}^{k} E_{j} E_{i}^{k^{\prime}}=0, \quad \text { if } i \neq j, \\
& \sum_{k+k^{\prime}=1-a_{i j}}(-1)^{k}\left[\begin{array}{c}
1-a_{i j} \\
k
\end{array}\right]_{v_{i}} s_{j i}^{k} p_{j i}^{-k} v_{i}^{k a_{i j}} F_{i}^{k^{\prime}} F_{j} F_{i}^{k}=0, \quad \text { if } i \neq j .
\end{aligned}
$$

By (10) and the assumption $s_{i j}=s_{j i}$, we have

$$
p_{i j}=p_{j i} s_{j i}^{-2}, \quad \forall i, j \in I .
$$

Let $U_{\mathbf{s}, \mathbf{p}}$ be the multi-parameter quantum superalgebra defined in $\mathrm{KKO}$, Section 2] associated to $(I, \cdot)$ which is a bar-consistent super Cartan datum. Let $\mathcal{J}$ be the ideal of $\mathbf{U}_{\mathbf{s}, \mathbf{p}}$ generated by $\left\{K_{i} K_{i}^{\prime} J_{i} J_{i}^{\prime}-1, \forall i \in I\right\}$, and $\mathbf{U}_{\mathbf{s}, \mathbf{p}}^{s q}$ the subalgebra of $\mathbf{U}_{\mathbf{s}, \mathbf{p}} / \mathcal{J}$ generated by $E_{i}, F_{i},\left(K_{i}^{\prime} J_{i}\right)^{ \pm}$and $\left(K_{i} J_{i}^{\prime}\right)^{ \pm}, \forall i \in I$.

Proposition 8. Under the assumptions (11) and $s_{i j}=s_{j i}$, the map $\Gamma$ : $U_{\mathbf{s}, \mathbf{p}} \rightarrow \mathbf{U}_{\mathbf{s}, \mathbf{p}}^{s q}$ sending $e_{i} \mapsto E_{i}, f_{i} \mapsto F_{i}, K_{i} \mapsto-h_{i} K_{i}^{\prime} J_{i}$ for all $i \in I$ is a $\mathbb{Q}$-algebra isomorphism.

4.5. The entire Multi-parameter quantum superalgebras II. In the case of the multi-parameter quantum superalgebra (see Example 5i), we further assume that $\tilde{s}_{i j}=\tilde{s}_{j i}$. Then, we set

$$
\xi(i, j)=\tilde{s}_{i j}^{-1} \quad \text { and } \quad \beta(i, j)=\tilde{s}_{i j} \tilde{p}_{i}^{a_{i j} / 2}, \forall i, j \in I .
$$

Let $\mathcal{G}(\nu)=\prod_{i, j \in I} \tilde{s}_{i j}^{-\nu(i) \nu(j) / 2}$, for all $\nu \in \mathbb{N}[I]$ (i.e., $\left.\nu=\sum_{i} \nu(i) i\right)$, then (26) holds.

Under these settings, we obtain an entire multi-parameter quantum superalgebra $\mathbf{U}_{\tilde{\mathbf{s}}, \tilde{\mathbf{p}}}$, generated by symbols $E_{i}, F_{i}, K_{i}^{ \pm 1}, K_{i}^{\prime \pm 1}, J_{i}^{ \pm 1}, J_{i}^{\prime \pm 1}, \forall i \in I$, and subjects to the following relations.

$$
\begin{array}{ll}
\left(R_{5} 1\right) & J_{i}^{ \pm 1}, J_{i}^{\prime \pm 1}, K_{i}^{ \pm 1} \text { and } K_{i}^{\prime \pm 1} \text { commute with each other, } \\
& K_{i}^{ \pm 1} K_{i}^{\mp 1}=K_{i}^{ \pm 1} K_{i}^{\prime \mp 1}=J_{i}^{ \pm 1} J_{i}^{\mp 1}=J_{i}^{\prime \pm 1} J_{i}^{\prime \mp 1}=1 . \\
\left(R_{5} 2\right) \quad & K_{i} E_{j}=E_{j} K_{i}, \quad K_{i}^{\prime} E_{j}=\tilde{s}_{i j} \tilde{p}_{i}^{a_{i j}} E_{j} K_{i}^{\prime}, \\
& K_{i} F_{j}=F_{j} K_{i}, \quad K_{i}^{\prime} F_{j}=\tilde{s}_{i j}^{-1} \tilde{p}_{i}^{-a_{i j}} F_{j} K_{i}^{\prime}, \\
& J_{i} E_{j}=\tilde{s}_{j i}^{-1} E_{j} J_{i}, \quad J_{i}^{\prime} E_{j}=E_{j} J_{i}^{\prime}, \\
& J_{i} F_{j}=\tilde{s}_{j i} F_{j} J_{i}, \quad J_{i}^{\prime} F_{j}=F_{j} J_{i}^{\prime} . \\
\left(R_{5} 3\right) \quad E_{i} F_{j}-\tilde{s}_{j i} F_{j} E_{i}=\delta_{i j} \frac{K_{i} J_{i}^{\prime}-J_{i} K_{i}^{\prime}}{v_{i}-v_{i}^{-1}} . \\
\left(R_{5} 4\right) \quad \sum_{k+k^{\prime}=1-a_{i j}}(-1)^{k}\left[\begin{array}{c}
1-a_{i j} \\
k
\end{array}\right]_{v_{i}}\left(\tilde{s}_{i j} \tilde{p}_{i}^{a_{i j} / 2}\right)^{-k} E_{i}^{k} E_{j} E_{i}^{k^{\prime}}=0, \quad \text { if } i \neq j,
\end{array}
$$




$$
\sum_{k+k^{\prime}=1-a_{i j}}(-1)^{k}\left[\begin{array}{c}
1-a_{i j} \\
k
\end{array}\right]_{v_{i}}\left(\tilde{s}_{i j} \tilde{p}_{i}^{a_{i j} / 2}\right)^{-k} F_{i}^{k^{\prime}} F_{j} F_{i}^{k}=0, \quad \text { if } i \neq j .
$$

Let $U_{\tilde{\mathbf{s}}, \tilde{\mathbf{p}}}$ be the multi-parameter quantum superalgebra defined in $\mathrm{KKO}$, Section 3] associated to $(I, \cdot)$ which is a bar-consistent super Cartan datum.

Let $\mathcal{L}$ be the ideal of $\mathbf{U}_{\tilde{\mathbf{s}}, \tilde{\mathbf{p}}}$ generated by $\left\{K_{i} J_{i}^{\prime}+v_{i}^{-1}, \forall i \in I\right\}$, and $\mathbf{U}_{\tilde{\mathbf{s}}, \tilde{\mathbf{p}}}^{s q}$ the subalgebra of $\mathbf{U}_{\tilde{\mathbf{s}}, \tilde{\mathbf{p}}} / \mathcal{L}$ generated by $E_{i}, F_{i}$ and $\left(K_{i}^{\prime} J_{i}\right)^{ \pm}, \forall i \in I$.

Proposition 9. Under the assumptions (13) and $\tilde{s}_{i j}=\tilde{s}_{j i}$, the map $\sigma$ : $U_{\tilde{\mathbf{s}}, \tilde{\mathbf{p}}} \rightarrow \mathbf{U}_{\tilde{\mathbf{s}}, \tilde{\mathbf{p}}}^{s q}$ sending $e_{i} \mapsto E_{i}, f_{i} \mapsto F_{i}$ and $\tilde{K}_{i} \mapsto-v_{i} K_{i}^{\prime} J_{i}$ for all $i \in I$ is a $\mathbb{Q}$-algebra isomorphism.

\section{REFERENCES}

[BGH] N. Bergeron, Y. Gao, and N. Hu, Drinfel'd doubles and Lusztig's symmetries of two-parameter quantum groups. Journal of Algebra, 2005, 301(1):378-405.

[BW] G. Benkart and S. Witherspoon, Two-parameter quantum groups and Drinfel'd doubles. Algebras \& Representation Theory, 2004, 7(3):261-286.

[C17] S Clark, Odd knot invariants from quantum covering groups. Algebraic \& Geometric Topology, 2017, 17(5):2961-3005.

[CFLW] S. Clark, Z. Fan, Y. Li, and W. Wang, Quantum supergroups III. Twistors. Communications in Mathematical Physics, 2014, 332(1):415-436.

[CHW] S. Clark, D. Hill, and W. Wang, Quantum supergroups I. foundations. Transformation Groups, 2013, 18(4):1019-1053.

[D87] V.G. Drinfeld, Quantum groups. In Proc. Int. Cong. Math. (Berkeley, 1986), pages 798-820. Amer. Math. Soc., Providence, RI, 1987. MR 89f:17017.

[FL] Z. Fan and Y. Li, Two-parameter quantum algebras, canonical bases and categorifications. International Mathematics Research Notices, 2015, 2015(16):7016-7062.

[FLL] Z. Fan, Y. Li, and Z. Lin, An identification of Lusztig's modified forms of quantum algebras and their analogues. 2013, arXiv:1312.0838

[GHZ] Y. Gao, N. Hu, and H. Zhang, Two-parameter quantum affine algebra of type $G_{2}^{(1)}$, Drinfeld realization and vertex representation. Journal of Mathematical Physics, 2015, 56(1):619-634.

[HPR] N. Hu, Y. Pei, and M. Rosso, Multi-parameter quantum groups and quantum shuffles. I. Quantum affine algebras, extended affine Lie algebras, and their applications, 2010, 506:145-171.

[HRZ] N. Hu, M. Rosso, and H. Zhang, Two-parameter quantum affine algebra $U_{r, s}\left(\widehat{\mathfrak{s l}_{n}}\right)$, Drinfel'd realization and quantum affine Lyndon basis. Communications in Mathematical Physics, 2008, 278(2):453-486.

[HZ] N. Hu and H. Zhang, Two-parameter quantum affine algebra of type $C_{n}^{(1)}$, Drinfeld realization and vertex representation. Journal of Algebra, 2016, 459:43-75.

[KKO] S.-J. Kang, M. Kashiwara, and S.-J. Oh, Supercategorification of quantum KacMoody algebras II. Advances in Mathematics, 2014, 265(1):169-240.

[L10] G. Lusztig, Introduction to quantum groups. Modern Birkhäuser Classics, Birkhäuser/Springer, New York, 2010.

[X97] J. Xiao, Drinfeld double and Ringel-Green Theory of Hall algebras. Journal of Algebra, 1997, 190(1):100-144.

College of Automation and College of Science, Harbin Engineering UniVERSiTy, HARBIn 150001, ChINA

E-mail address: fanz@math.ksu.edu (Fan) 
College of Automation, Harbin Engineering University, Harbin 150001, CHINA

E-mail address: xingjunjing2017@hrbeu.edu.cn (Xing) 